\title{
Case Study 1
}

\section{Unoriented Bordism}

A simple observation about the bordism ring $M O_{*}(*)$ (or $M O_{*}(X)$ more generally, for any space $X$ ) is that it consists entirely of 2-torsion: Any chain $Z \rightarrow X$ can be bulked out to a constant cylinder $Z \times I \rightarrow X$, which has as its boundary the chain $2 \cdot(Z \rightarrow X)$. Accordingly, $M O_{*}(X)$ is always an $\mathbb{F}_{2}$-vector space. Our goal in this case study is to arrive at two remarkable calculations: First, in Corollary 1.5.7 we will make an explicit calculation of this $\mathbb{F}_{2}$-vector space in the case of the bordism homology of a point; and second, in Lemma 1.5.8 we will show that there is a natural isomorphism

$$
M O_{*}(X)=H \mathbb{F}_{2 *}(X) \otimes_{\mathbb{F}_{2}} M O_{*}(*) .
$$

Our goal in discussing these results in the first case study of the book is to take the opportunity to introduce several key concepts that will serve us throughout. First and foremost, we will require a definition of bordism spectrum that we can manipulate computationally, using just the tools of abstract homotopy theory. Once that is established, we immediately begin to bring algebraic geometry into the mix: The main idea is that the cohomology ring of a space is better viewed as a scheme (with plenty of extra structure), and the homology groups of a spectrum are better viewed as a representation for a certain elaborate algebraic group. This data actually finds familiar expression in homotopy theory: We show that a form of group cohomology for this representation forms the input to the classical Adams spectral sequence, which classically takes the form

$$
\operatorname{Cotor}_{\mathcal{A}_{*}}^{*, *}\left(\mathbb{F}_{2}, H \mathbb{F}_{2 *}(Y)\right) \Rightarrow \pi_{*}(Y),
$$

converging for certain very nice spectra $Y$-including, for instance, $Y=M O$. In particular, we can bring the tools from the preceding discussion to bear on the homology and cohomology of $M O$, where we make an explicit calculation of its representation structure. Finding that it is suitably free, we thereby gain 
control of the Adams spectral sequence, finish the computation, and prove the desired result.

Our real goal in this case study, however, is to introduce one of the main phenomena guiding this text: There is some governing algebro-geometric object, the formal group $\mathbb{R P}_{H \mathbb{F}_{2}}^{\infty}$, which exerts an extraordinary amount of control over everything in sight. We will endeavor to rephrase as much of this classical computation as possible so as to highlight its connection to this central object, and we will use this as motivation in future case studies to pursue similar objects, which will lead us down much deeper and more rewarding rabbit holes. The counterbalance to this is that, at least for now, we will not introduce concepts or theorems in their maximum generality. ${ }^{1}$ Essentially everything mentioned in this case study will be re-examined more thoroughly in future case studies, so the reader is advised to look to those for the more expansive set of results.

\subsection{Thom Spectra and the Thom Isomorphism}

Our goal is a sequence of theorems about the unoriented bordism spectrum $M O$. We will begin by recalling a definition of the spectrum $M O$ using just abstract homotopy theory, because it involves ideas that will be useful to us throughout the text and because we cannot compute effectively with the chain-level definition given in the Introduction.

Definition 1.1.1 For a spherical bundle $S^{n-1} \rightarrow \xi \rightarrow X$, its Thom space is given by the cofiber

$$
\xi \rightarrow X \stackrel{\text { cofiber }}{\longrightarrow} T_{n}(\xi) .
$$

"Proof" of definition There is a more classical construction of the Thom space: Take the associated disk bundle by gluing an $n$-disk fiberwise, and add a point at infinity by collapsing $\xi$ :

$$
T_{n}(\xi)=\left(\xi \cup_{X \times S^{n-1}}\left(X \times D^{n}\right)\right)^{+} .
$$

To compare this with the cofiber definition, recall that the thickening of $\xi$ to an $n$-disk bundle is the same as taking the mapping cylinder on $\xi \rightarrow X$. Since the inclusion into the mapping cylinder is now a cofibration, the quotient by this subspace agrees with both the cofiber of the map and the introduction of a point at infinity.

Before proceeding, here are two important examples:

\footnotetext{
${ }^{1}$ For an obvious example, everything in this case study will be done relative to $\operatorname{Spec} \mathbb{F}_{2}$.
} 
Example 1.1.2 If $\xi=S^{n-1} \times X$ is the trivial bundle, then $T_{n}(\xi)=S^{n} \wedge\left(X_{+}\right)$. This is supposed to indicate what Thom spaces are "doing": if you feed in the trivial bundle then you get the suspension out, so if you feed in a twisted bundle you should think of it as a twisted suspension.

Example 1.1.3 Let $\xi$ be the tautological $S^{0}$-bundle over the unpointed space $\mathbb{R} \mathrm{P}^{\infty}=B O(1)$. Because $\xi$ has contractible total space, $E O(1)$, the cofiber degenerates and it follows that $T_{1}(\xi)=\mathbb{R} \mathrm{P}^{\infty}$, a pointed space. ${ }^{2}$ More generally, arguing by cells shows that the Thom space for the tautological bundle over $\mathbb{R} \mathrm{P}^{n}$ is $\mathbb{R} \mathrm{P}^{n+1}$.

Now we catalog a bunch of useful properties of the Thom space construction. First, recall that a spherical bundle over $X$ is the same data [May75] as a map $X \rightarrow B G L_{1} S^{n-1}$, where $G L_{1} S^{n-1}$ is the subspace of $F\left(S^{n-1}, S^{n-1}\right)$ expressed by the pullback of spaces

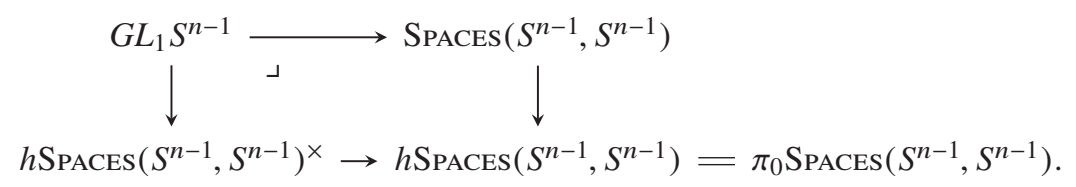

Lemma 1.1.4 The construction $T_{n}$ can be viewed as a functor from the slice category over $B G L_{1} S^{n-1}$ to SPACES. Maps of slices

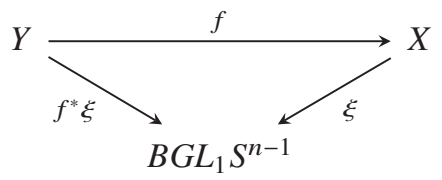

induce maps $T_{n}\left(f^{*} \xi\right) \rightarrow T_{n}(\xi)$, and $T_{n}$ is suitably homotopy-invariant.

Next, the spherical subbundle of a vector bundle gives a common source of spherical bundles. The action of $O(n)$ on $\mathbb{R}^{n}$ preserves the unit sphere, and hence gives a map $O(n) \rightarrow G L_{1} S^{n-1}$. These are maps of topological groups, and the block-inclusion maps $i^{n}: O(n) \rightarrow O(n+1)$ commute with the suspension map $G L_{1} S^{n-1} \rightarrow G L_{1} S^{n}$. In fact, much more can be said:

Lemma 1.1.5 The block-sum maps $O(n) \times O(m) \rightarrow O(n+m)$ are compatible with the join maps $G L_{1} S^{n-1} \times G L_{1} S^{m-1} \rightarrow G L_{1} S^{n+m-1}$.

Taking a cue from $K$-theory, we pass to the colimit as $n$ grows large, using the maps

$\overline{2}$ If you already know what's coming, this should comport with the Thom isomorphism, which asserts $x \cdot H \mathbb{F}_{2}^{*} \mathbb{R} P^{\infty} \cong{\widetilde{H \mathbb{F}_{2}}}^{*+1} \mathbb{R} \mathrm{P}^{\infty}$. 


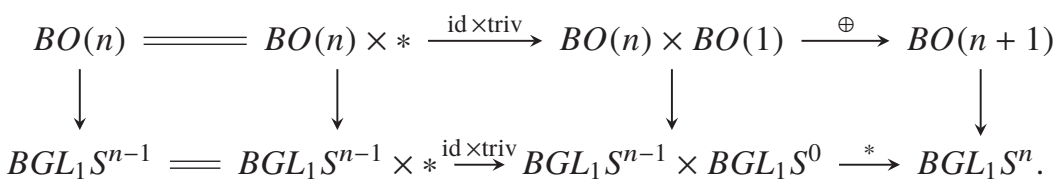

Corollary 1.1.6 The operations of block-sum and topological join imbue the colimiting spaces $B O$ and $B G L_{1} \mathbb{S}$ with the structure of $H$-groups. Moreover, the colimiting map

$$
J_{\mathbb{R}}: B O \rightarrow B G L_{1} \mathbb{S},
$$

called the J-homomorphism, is a morphism of H-groups.

Finally, we can ask about the compatibility of Thom constructions with all of this. In order to properly phrase the question, we need a version of the construction which operates on stable spherical bundles, i.e., whose source is the slice category over $B G L_{1} \mathbb{S}$. By calculating

$$
T_{n+1}(\xi * \operatorname{triv}) \simeq \Sigma T_{n}(\xi),
$$

we are inspired to make the following definition:

Definition 1.1.7 For $\xi$ an $S^{n-1}$-bundle, we define the Thom spectrum of $\xi$ to be

$$
T(\xi):=\Sigma^{-n} \Sigma^{\infty} T_{n}(\xi)
$$

By filtering the base space by compact subspaces, this begets a functor

$$
T: \operatorname{SPACES}_{/ B G L_{1} \mathbb{S}} \rightarrow \text { SPECTRA. }
$$

Lemma 1.1.8 $T$ is monoidal: It carries external fiberwise joins to smash products of Thom spectra. Correspondingly, $T \circ J_{\mathbb{R}}$ carries external direct sums of stable vector bundles to smash products of Thom spectra.

Definition 1.1.9 The spectrum $M O$ arises as the universal example of all these constructions, strung together:

$$
M O:=T\left(J_{\mathbb{R}}\right)=\underset{n}{\operatorname{colim}} T\left(J_{\mathbb{R}}^{n}\right)=\underset{n}{\operatorname{colim}} \Sigma^{-n} T_{n} J_{\mathbb{R}}^{n} .
$$

The spectrum $M O$ has several remarkable properties. First, it can be shown that the generalized homology theory that this spectrum encodes matches the one described in the Introduction [Swi02, theorem 12.30], [Rud98, theorem 7.27]. The most basic homotopical property is that this spectrum is naturally a ring spectrum, and this follows immediately from $J_{\mathbb{R}}$ being a homomorphism of $H$-spaces. Much more excitingly, we can also deduce the presence of Thom isomorphisms just from the properties stated thus far. That $J_{\mathbb{R}}$ is a homomorphism means that the square in the following diagram commutes: 


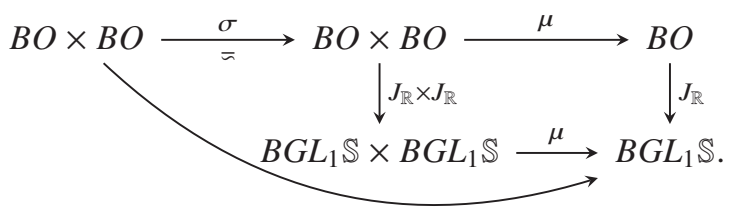

We have extended this square very slightly by a certain shearing map $\sigma$ defined by $\sigma(x, y)=\left(x y^{-1}, y\right)$. It is evident that $\sigma$ is a homotopy equivalence, since just as we can de-scale the first coordinate by $y$ we can re-scale by it - indeed, this is the observation that $B O$ is a torsor for itself. We can calculate directly the behavior of the long composite:

$$
J_{\mathbb{R}} \circ \mu \circ \sigma(x, y)=J_{\mathbb{R}} \circ \mu\left(x y^{-1}, y\right)=J_{\mathbb{R}}\left(x y^{-1} y\right)=J_{\mathbb{R}}(x) .
$$

It follows that the second coordinate plays no role, and that the bundle classified by the long composite can be written as $J_{\mathbb{R}} \times 0 .{ }^{3}$ We are now in a position to see the Thom isomorphism:

Lemma 1.1.10 (Thom isomorphism, universal example, see [Mah79]) As MO-modules,

$$
M O \wedge M O \simeq M O \wedge \Sigma_{+}^{\infty} B O .
$$

Proof Stringing together the naturality properties of the Thom functor outlined above, we can thus make the following calculation:

$$
\begin{aligned}
T\left(\mu \circ\left(J_{\mathbb{R}} \times J_{\mathbb{R}}\right)\right) & \simeq T\left(\mu \circ\left(J_{\mathbb{R}} \times J_{\mathbb{R}}\right) \circ \sigma\right) \\
& \simeq T\left(\mu \circ\left(J_{\mathbb{R}} \times 0\right)\right) \\
& \simeq T\left(J_{\mathbb{R}}\right) \wedge T(0) \\
& \simeq T\left(J_{\mathbb{R}}\right) \wedge \Sigma_{+}^{\infty} B O \\
T\left(J_{\mathbb{R}}\right) \wedge T\left(J_{\mathbb{R}}\right) & \simeq T\left(J_{\mathbb{R}}\right) \wedge \Sigma_{+}^{\infty} B O \\
M O \wedge M O & \simeq M O \wedge \Sigma_{+}^{\infty} B O .
\end{aligned}
$$

(homotopy invariance) (constructed lift) (monoidality) (monoidality) (definition of $M O$ )

In order to verify that this equivalence is one of $M O$-modules, one performs an analogous computation with $J_{\mathbb{R}}^{\times 3}$.

From here, the general version of Thom's theorem follows quickly:

Definition 1.1.11 A map $\varphi: M O \rightarrow E$ of homotopy ring spectra is called a (real) orientation of $E$ (by $M O$ ).

\footnotetext{
3 This factorization does not commute with the rest of the diagram, just with the little lifting triangle it forms.
} 
Theorem 1.1.12 (Thom isomorphism) Let $\xi: X \rightarrow B O$ classify a vector bundle and let $\varphi: M O \rightarrow E$ be a map of ring spectra. Then there is an equivalence of $E$-modules

$$
E \wedge T(\xi) \simeq E \wedge \Sigma_{+}^{\infty} X .
$$

Modifications to above proof To accommodate $X$ rather than $B O$ as the base, we redefine $\sigma: B O \times X \rightarrow B O \times X$ by

$$
\sigma(x, y)=\sigma\left(x \xi(y)^{-1}, y\right) .
$$

Follow the same proof as before with the diagram

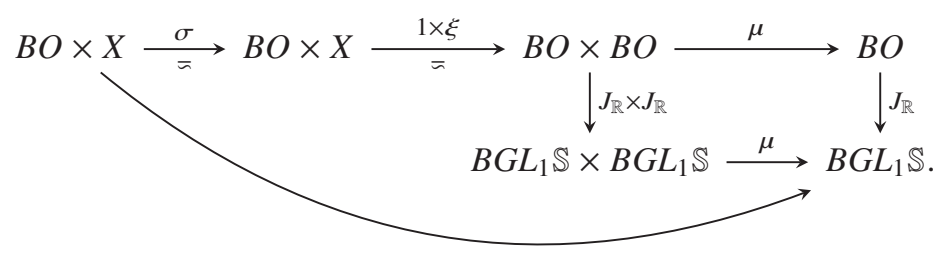

This gives an equivalence $\theta_{M O}: M O \wedge T(\xi) \rightarrow M O \wedge \Sigma_{+}^{\infty} X$ of $M O$-modules. To introduce $E$, note that there is a diagram of $M O$-module spectra

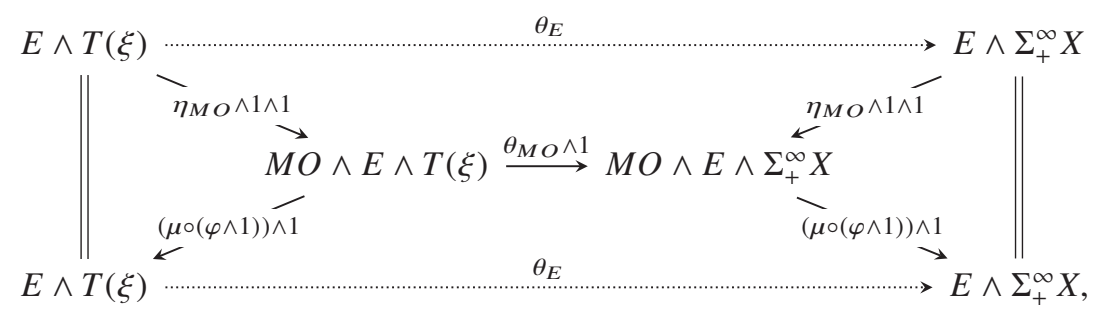

whose columns are retractions. The dotted arrows $\theta_{E}$ are defined by following the solid path from corner to corner. Similarly, the inverse $\alpha_{M O}$ to $\theta_{M O}$ induces a map $\alpha_{E}$. We claim that $\theta_{E}$ and $\alpha_{E}$ are themselves inverses, which is shown by staggering and rearranging where the collapse of the $M O$ factor into $E$ happens. The composite $\alpha_{E} \circ \theta_{E}$ is equivalent to the following:

$$
\begin{aligned}
E \wedge \Sigma_{+}^{\infty} & X \stackrel{1 \wedge \eta \wedge 1}{\longrightarrow} E \wedge M O \wedge \Sigma_{+}^{\infty} X \\
& \stackrel{1 \wedge 1 \wedge \eta \wedge 1}{\longrightarrow} E \wedge M O \wedge M O \wedge \Sigma_{+}^{\infty} X \\
& \stackrel{1 \wedge 1 \wedge \theta_{M O}}{\longrightarrow} E \wedge M O \wedge M O \wedge T(\xi) \\
& \stackrel{1 \wedge \tau \wedge 1}{\longrightarrow} E \wedge M O \wedge M O \wedge T(\xi)
\end{aligned}
$$




$$
\begin{aligned}
& \stackrel{1 \wedge 1 \wedge \alpha_{M O}}{\longrightarrow} E \wedge M O \wedge M O \wedge \Sigma_{+}^{\infty} X \\
& \stackrel{\mu \wedge 1 \wedge 1}{\longrightarrow} E \wedge M O \wedge \Sigma_{+}^{\infty} X \\
& \stackrel{\mu \wedge 1}{\longrightarrow} E \wedge \Sigma_{+}^{\infty} X,
\end{aligned}
$$

where $\tau$ is the twist map. Using the commuting square

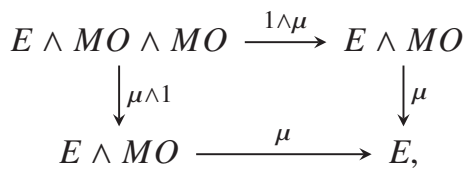

we have that the middle composite is equivalent to

$$
\begin{aligned}
E \wedge \Sigma_{+}^{\infty} X \stackrel{1 \wedge \eta \wedge 1}{\longrightarrow} E \wedge M O \wedge \Sigma_{+}^{\infty} X \\
\stackrel{1 \wedge \theta_{M O}}{\longrightarrow} E \wedge M O \wedge T(\xi) \\
\stackrel{1 \wedge \alpha_{M O}}{\longrightarrow} E \wedge M O \wedge \Sigma_{+}^{\infty} X \\
\stackrel{\mu \wedge 1}{\longrightarrow} E \wedge \Sigma_{+}^{\infty} X,
\end{aligned}
$$

which is the identity on $E \wedge \Sigma_{+}^{\infty} X$. The proof that $\alpha_{E}$ is also the right-inverse of $\theta_{E}$ is similar.

Remark 1.1.13 One of the tentpoles of the theory of Thom spectra is that Theorem 1.1.12 has a kind of converse: If a ring spectrum $E$ has suitably natural and multiplicative Thom isomorphisms for Thom spectra formed from real vector bundles, then one can define an essentially unique ring map $M O \rightarrow E$ that realizes these isomorphisms via the machinery of Theorem 1.1.12.

Remark 1.1.14 There is also a cohomological version of the Thom isomorphism. Suppose that $E$ is a ring spectrum under $M O$ and let $\xi$ be the spherical bundle of a vector bundle on a space $X$. The spectrum $F\left(\Sigma_{+}^{\infty} X, E\right)$ is a ring spectrum under $E$ (hence under $M O$ ), so there is a Thom isomorphism as well as an evaluation map

$$
F\left(\Sigma_{+}^{\infty} X, E\right) \wedge T(\xi) \stackrel{\simeq}{\rightarrow} F\left(\Sigma_{+}^{\infty} X, E\right) \wedge \Sigma_{+}^{\infty} X \stackrel{\text { eval }}{\longrightarrow} E .
$$

Passing through the exponential adjunction, the map

$$
F\left(\Sigma_{+}^{\infty} X, E\right) \stackrel{\simeq}{\rightarrow} F(T(\xi), E)
$$

can be seen to give the cohomological Thom isomorphism

$$
E^{*} X \cong E^{*} T(\xi)
$$


Example 1.1.15 We will close out this section by using this to actually make a calculation. Recall from Example 1.1.3 that $T_{1} \circ J_{\mathbb{R}}\left(\mathcal{L}\right.$ over $\left.\mathbb{R} P^{n}\right)$ is given by $\mathbb{R} \mathrm{P}^{n+1}$. Because $M O$ is a connective spectrum, the truncation map

$$
M O \rightarrow M O(-\infty, 0]=H \pi_{0} M O=H \mathbb{F}_{2}
$$

is a map of ring spectra [May77, lemma II.2.12]. Hence, we can apply the Thom isomorphism theorem to the mod-2 homology of Thom complexes coming from real vector bundles:

$$
\begin{array}{rlr}
\pi_{*}\left(H \mathbb{F}_{2} \wedge T(\mathcal{L}-1)\right) & \cong \pi_{*}\left(H \mathbb{F}_{2} \wedge T(0)\right) & \text { (Thom isomorphism) } \\
\pi_{*}\left(H \mathbb{F}_{2} \wedge \Sigma^{-1} \Sigma^{\infty} \mathbb{R P}^{n+1}\right) & \cong \pi_{*}\left(H \mathbb{F}_{2} \wedge \Sigma_{+}^{\infty} \mathbb{R} P^{n}\right) & \text { (Example 1.1.3) } \\
\widetilde{H \mathbb{F}}_{2 *+1} \mathbb{R P}^{n+1} & \cong H \mathbb{F}_{2 *} \mathbb{R P}^{n} . & \text { (generalized homology) }
\end{array}
$$

This powers an induction that shows that $H \mathbb{F}_{2 *} \mathbb{R} P^{\infty}$ has a single class in every degree. The cohomological version of the Thom isomorphism in Remark 1.1.14, together with the $H \mathbb{F}_{2}^{*} \mathbb{R} P^{n}$-module structure of $H \mathbb{F}_{2}^{*} T(\mathcal{L}-1)$, also gives the ring structure:

$$
H \mathbb{F}_{2}^{*} \mathbb{R} \mathrm{P}^{n}=\mathbb{F}_{2}[x] / x^{n+1} .
$$

\subsection{Cohomology Rings and Affine Schemes}

An abbreviated summary of this book is that we are going to put "Spec" in front of rings appearing in algebraic topology and see what happens. Before actually doing any algebraic topology, we should recall what this means on the level of algebra. The core idea is to replace an $\mathbb{F}_{2}$-algebra $A$ by the functor it corepresents, which we will denote by $\operatorname{Spec} A$. For any other "test $\mathbb{F}_{2}$-algebra" $T$, we set

$(\operatorname{Spec} A)(T):=\operatorname{AlgebraS}_{\mathbb{F}_{2} /}(A, T) \cong \operatorname{SCHEMES}_{\mathbb{F}_{2}}(\operatorname{Spec} T, \operatorname{Spec} A)$.

More generally, we have the following definition:

Definition 1.2.1 An affine $\mathbb{F}_{2}$-scheme is a functor $X:$ Algebras $_{\mathbb{F}_{2} /} \rightarrow$ Sets which is (noncanonically) isomorphic to Spec $A$ for some $\mathbb{F}_{2}$-algebra $A$. Given such an isomorphism, we will refer to $\operatorname{Spec} A \rightarrow X$ as a parameter for $X$ and its inverse $X \rightarrow \operatorname{Spec} A$ as a coordinate for $X$.

Lemma 1.2.2 There is an equivalence of categories

$$
\text { Spec : } \operatorname{AlgEBRAS}_{\mathbb{F}_{2} /}^{\mathrm{op}} \rightarrow \operatorname{AFFINESCHEMES}_{/ \mathbb{F}_{2}} .
$$


The centerpiece of thinking about rings in this way, for us and for now, is to translate between a presentation of $A$ as a quotient of a free algebra and a presentation of $(\operatorname{Spec} A)(T)$ as selecting tuples of elements in $T$ subject to certain conditions. Consider the following example:

Example 1.2.3 Set $A_{n}=\mathbb{F}_{2}\left[x_{1}, \ldots, x_{n}\right]$. Elements of $\left(\operatorname{Spec} A_{n}\right)(T)$ can be identified with $n$-tuples of elements of $T$, since a function in

$$
\left(\operatorname{Spec} A_{n}\right)(T)=\operatorname{AlgEBRAS}_{\mathbb{F}_{2} /}\left(\mathbb{F}_{2}\left[x_{1}, \ldots, x_{n}\right], T\right)
$$

is entirely determined by where the $x_{j}$ are sent. Consider also what happens when we impose a relation by passing to $A_{n}^{J}=\mathbb{F}_{2}\left[x_{1}, \ldots, x_{n}\right] /\left(x_{k}^{j_{k}+1}\right)$ : a function in

$$
\left(\operatorname{Spec} A_{n}^{J}\right)(T)=\operatorname{AlgEBRAS}_{\mathbb{F}_{2} /}\left(\mathbb{F}_{2}\left[x_{1}, \ldots, x_{n}\right] /\left(x_{k}^{j_{k}+1}\right), T\right)
$$

is again determined by where the $x_{j}$ are sent, but now $x_{j}$ can only be sent to an element which is nilpotent of order $j_{k}+1$. These schemes are both important enough that we give them special names:

$$
\mathbb{A}^{n}:=\operatorname{Spec} \mathbb{F}_{2}\left[x_{1}, \ldots, x_{n}\right], \quad \mathbb{A}^{n, J}:=\operatorname{Spec} \mathbb{F}_{2}\left[x_{1}, \ldots, x_{n}\right] /\left(x_{k}^{j_{k}+1}\right) .
$$

The functor $\mathbb{A}^{n}$ is called affine $n$-space - reasonable, since the value $\mathbb{A}^{n}(T)$ is isomorphic to $T^{n}$. We refer to $\mathbb{A}^{1}$ as the affine line. Note that the quotient map $A_{1} \rightarrow A_{1}^{(j)}$ induces an inclusion $\mathbb{A}^{1,(j)} \rightarrow \mathbb{A}^{1}$ and that $\mathbb{A}^{1,(0)}$ is a constant functor:

$$
\mathbb{A}^{1,(0)}(T)=\left\{f: \mathbb{F}_{2}[x] \rightarrow T \mid f(x)=0\right\}
$$

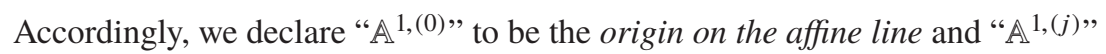
to be the $(n+1)$ st order (nilpotent) neighborhood of the origin in the affine line.

We can also use this language to re-express another common object arising in algebraic topology: the Hopf algebra, which appears when taking the mod-2 cohomology of an $H$-group. In addition to the usual ring structure on cohomology groups, the $H$-group multiplication, unit, and inversion maps additionally induce a diagonal map $\Delta$, an augmentation map $\varepsilon$, and an antipode $\chi$, respectively. Running through the axioms, one quickly checks the following:

Lemma 1.2.4 For a Hopf $\mathbb{F}_{2}$-algebra A, the functor $\mathrm{Spec} A$ is naturally valued in groups. Such functors are called group schemes. Conversely, a choice of group structure on Spec A endows A with the structure of a Hopf algebra.

Proof sketch This is a matter of recognizing the product in ALgEBRAs $\mathrm{F}_{\mathbb{F}_{2} /}^{\mathrm{op}}$ as the tensor product, then using the Yoneda lemma to transfer the structure around. 
Example 1.2.5 The functor $\mathbb{A}^{1}$ previously introduced is naturally valued in groups: Since $\mathbb{A}^{1}(T) \cong T$, we can use the addition on $T$ to make it into an abelian group. When considering $\mathbb{A}^{1}$ with this group scheme structure, we denote it as $\mathbb{G}_{a}$. Applying the Yoneda lemma, one deduces the following formulas for the Hopf algebra structure maps: ${ }^{4}$

$$
\begin{array}{rlr}
\mathbb{G}_{a} \times \mathbb{G}_{a} \stackrel{\mu}{\rightarrow} \mathbb{G}_{a} & x_{1}+x_{2} & \leftrightarrow x, \\
\mathbb{G}_{a} \stackrel{\chi}{\rightarrow} \mathbb{G}_{a} & -x & \leftrightarrow x, \\
\operatorname{Spec} \mathbb{F}_{2} \stackrel{\eta}{\rightarrow} \mathbb{G}_{a} & 0 & \leftrightarrow x,
\end{array}
$$

where we have written $x_{1}=x \otimes 1$ and $x_{2}=1 \otimes x$ for the elements of

$$
O_{\mathbb{G}_{a} \times \mathbb{G}_{a}} \cong O_{\mathbb{G}_{a}} \otimes O_{\mathbb{G}_{a}} \cong \mathbb{F}_{2}[x] \otimes \mathbb{F}_{2}[x] .
$$

As an example of how to reason this out, consider the following diagram:

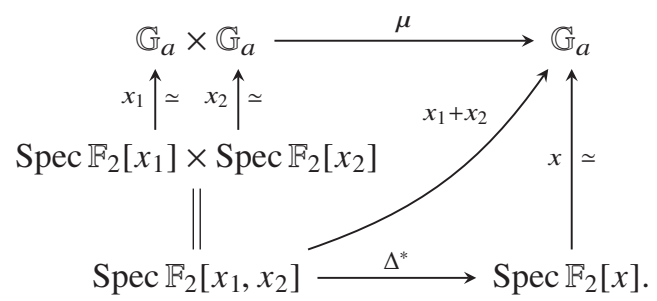

It follows that the bottom map of affine schemes is induced by the algebra map

$$
\mathbb{F}_{2}[x] \stackrel{\Delta}{\rightarrow} \mathbb{F}_{2}\left[x_{1}, x_{2}\right], \quad x \mapsto x_{1}+x_{2} .
$$

Remark 1.2.6 In fact, $\mathbb{A}^{1}$ is naturally valued in rings. It models the inverse functor to Spec in the equivalence of these categories, i.e., the elements of a ring $A$ always form a complete collection of $\mathbb{A}^{1}$-valued functions on some affine scheme $\operatorname{Spec} A$.

Example 1.2.7 We define the multiplicative group scheme by

$$
\mathbb{G}_{m}=\operatorname{Spec} \mathbb{F}_{2}[x, y] /(x y-1) .
$$

Its value $\mathbb{G}_{m}(T)$ on a test algebra $T$ is the set of pairs $(x, y)$ such that $y$ is a multiplicative inverse to $x$, and hence $\mathbb{G}_{m}$ is valued in groups. Applying the Yoneda lemma, we deduce the following formulas for the Hopf algebra structure maps:

4 Of course, since we are working over $\mathbb{F}_{2}$, we could just as well write $\chi(x)=x$. 


$$
\begin{array}{rl}
\mathbb{G}_{m} \times \mathbb{G}_{m} \stackrel{\mu}{\rightarrow} \mathbb{G}_{m} & x_{1} \otimes x_{2} \leftrightarrow x \\
y_{1} \otimes y_{2} & \leftrightarrow y, \\
\mathbb{G}_{m} \stackrel{x}{\rightarrow} \mathbb{G}_{m} & (y, x) \leftrightarrow(x, y), \\
\operatorname{Spec} \mathbb{F}_{2} \stackrel{\eta}{\rightarrow} \mathbb{G}_{m} & 1 \leftrightarrow x, y .
\end{array}
$$

Remark 1.2.8 As presented earlier the multiplicative group comes with a natural inclusion $\mathbb{G}_{m} \rightarrow \mathbb{A}^{2}$. Specifically, the subset $\mathbb{G}_{m} \subseteq \mathbb{A}^{2}$ consists of pairs $(x, y)$ in the graph of the hyperbola $y=1 / x$. However, the element $x$ also gives an $\mathbb{A}^{1}$-valued function $x: \mathbb{G}_{m} \rightarrow \mathbb{A}^{1}$, and because multiplicative inverses in a ring are unique, we see that this map too is an inclusion. These two inclusions have rather different properties relative to their ambient spaces, and we will think harder about these essential differences later on.

Example 1.2.9 (see Example 4.4.12) This example showcases the complications that algebraic geometry introduces to this situation, and is meant as discouragement from thinking of the theory of affine group schemes as a strong analog of the theory of linear complex Lie groups. It jumps ahead of the present narrative by a fair amount - the reader should feel completely comfortable skipping this example for now. We set $\alpha_{2}=\operatorname{Spec} \mathbb{F}_{2}[x] /\left(x^{2}\right)$, with group scheme structure given by

$$
\begin{array}{rlr}
\alpha_{2} \times \alpha_{2} \stackrel{\mu}{\rightarrow} \alpha_{2} & x_{1}+x_{2} & \leftrightarrow x, \\
\alpha_{2} \stackrel{\chi}{\rightarrow} \alpha_{2} & -x & \leftrightarrow x, \\
\text { Spec } \mathbb{F}_{2} \stackrel{\eta}{\rightarrow} \alpha_{2} & 0 \leftrightarrow x .
\end{array}
$$

This group scheme has several interesting properties which we will merely state, reserving their proofs for Example 4.4.12.

1. $\alpha_{2}$ has the same underlying structure ring as $\mu_{2}:=\mathbb{G}_{m}[2]$, the 2-torsion points of $\mathbb{G}_{m}$, but is not isomorphic to it.

2. There is no commutative group scheme $G$ of rank four such that $\alpha_{2}=G[2]$.

3. If $E / \mathbb{F}_{2}$ is the supersingular elliptic curve, then there is a short exact sequence

$$
0 \rightarrow \alpha_{2} \rightarrow E[2] \rightarrow \alpha_{2} \rightarrow 0 .
$$

However, this short exact sequence does not split (even after base change).

4. The subgroups of $\alpha_{2} \times \alpha_{2}$ of order 2 are parameterized by the scheme $\mathbb{P}^{1}$, i.e., for $A$ an $\mathbb{F}_{2}$-algebra the subgroup schemes of $\alpha_{2} \times \alpha_{2}$ of order 2 which are defined over $A$ are parameterized by the set $\mathbb{P}^{1}(A)$.

We now turn to a different class of examples, which will wind up being the key players in our upcoming topological story. To begin, consider the colimit of the sets $\operatorname{colim}_{j \rightarrow \infty} \mathbb{A}^{1,(j)}(T)$, which is of use in algebra: it is the collection of 
nilpotent elements in $T$. These kinds of conditions that are "unbounded in $j$ " appear frequently enough that we are moved to give these functors a name too:

Definition 1.2.10 An affine formal scheme is an ind-system of finite affine schemes. The morphisms between two formal schemes are computed ${ }^{5,6}$ by

$$
\text { Formal Schemes }\left(\left\{X_{\alpha}\right\},\left\{Y_{\beta}\right\}\right)=\lim _{\alpha} \operatorname{colim} \operatorname{Schemes}\left(X_{\alpha}, Y_{\beta}\right) \text {. }
$$

Given affine charts $X_{\alpha}=\operatorname{Spec} A_{\alpha}$, we will glibly suppress the system from the notation and write

$$
\operatorname{Spf} A:=\left\{\operatorname{Spec} A_{\alpha}\right\} .
$$

Example 1.2.11 The individual schemes $\mathbb{A}^{1,(j)}$ do not support group structures. After all, the sum of two elements which are nilpotent of order $j+1$ can only be guaranteed to be nilpotent of order $2 j+1$. It follows that the entire ind-system $\left\{\mathbb{A}^{1,(j)}\right\}=: \widehat{\mathbb{A}}^{1}$ supports a group structure, even though none of its constituent pieces do. We call such an object a formal group scheme, and this particular formal group scheme we denote by $\widehat{\mathbb{G}}_{a}$.

Example 1.2.12 Similarly, one can define the scheme $\mathbb{G}_{m}[j]$ of elements of unipotent order $j$ :

$$
\mathbb{G}_{m}[j]=\operatorname{Spec} \frac{\mathbb{F}_{2}[x, y]}{\left(x y-1, x^{j}-1\right)} \subseteq \mathbb{G}_{m} .
$$

These are all group schemes, and they nest together in a complicated way: There is an inclusion of $\mathbb{G}_{m}[j]$ into $\mathbb{G}_{m}[j k]$. There is also a second filtration along the lines of the one considered in Example 1.2.11:

$$
\mathbb{G}_{m}^{(j)}=\operatorname{Spec} \frac{\mathbb{F}_{2}[x, y]}{\left(x y-1,(x-1)^{j}\right)} .
$$

These schemes form a sequential system, but they are only occasionally group

5 For the categorical reader, we include a significant categorical aside: Passing to ind-systems has the effect of formally adjoining colimits of filtered diagrams to a category. The formula for the mapping set comes from asserting that the assignment $\mathrm{C} \rightarrow \operatorname{Ind}(\mathrm{C})$ is fully faithful, that its image consists of compact objects, and that each diagram $\mathrm{D} \rightarrow \mathrm{C}$ which can be interpreted as a member of $\operatorname{Ind}(\mathrm{C})$ is its own colimit. To control the difference between this category and the original category, it is often useful to restrict attention to diagrams of objects which are already compact in $\mathrm{C}$, as in our definition of a formal scheme.

6 Along the same lines, one can show the following recognition principle [Str99b, proposition 4.6]: a functor $X:$ Algebras $\rightarrow$ SETS which preserves finite limits is a formal scheme exactly when there exists a family of maps $X_{i} \rightarrow X$ from a set of affine schemes $X_{i}$ such that for all test algebras $T$ the following map is onto:

$$
\coprod_{i} X_{i}(T) \rightarrow X(T) .
$$


schemes. Specifically, $\mathbb{G}_{m}^{\left(2^{k}\right)}$ is a group scheme, in which case $\mathbb{G}_{m}^{\left(2^{k}\right)} \cong \mathbb{G}_{m}\left[2^{k}\right]{ }^{7}$ We define $\widehat{\mathbb{G}}_{m}$ using this common subsystem:

$$
\widehat{\mathbb{G}}_{m}:=\left\{\mathbb{G}_{m}^{\left(2^{k}\right)}\right\}_{k=0}^{\infty} \text {. }
$$

Let us now consider the example that we closed with in the previous Lecture, where we calculated $H \mathbb{F}_{2}^{*}\left(\mathbb{R P}^{n}\right)=\mathbb{F}_{2}[x] /\left(x^{n+1}\right)$. Putting "Spec" in front of this, we could reinterpret this calculation as

$$
\operatorname{Spec} H \mathbb{F}_{2}^{*}\left(\mathbb{R} P^{n}\right) \cong \mathbb{A}^{1,(n)}
$$

This is so useful that we will give it a notation all of its own:

Definition 1.2.13 Let $X$ be a finite cell complex, so that $\operatorname{HF}_{2}^{*}(X)$ is a ring which is finite dimensional as an $\mathbb{F}_{2}$-vector space. We will write

$$
X_{H \mathbb{F}_{2}}=\operatorname{Spec} H \mathbb{F}_{2}^{*} X
$$

for the corresponding finite affine scheme.

Example 1.2.14 Putting together the discussions from this Lecture and the previous one, in the new notation we have calculated

$$
\mathbb{R P}_{H \mathbb{F}_{2}}^{n} \cong \mathbb{A}^{1,(n)} \text {. }
$$

So far, this example just restates what we already knew in a mildly different language. Our driving goal for the next section is to incorporate as much information as we have about these cohomology rings $H \mathbb{F}_{2}^{*}\left(\mathbb{R P}^{n}\right)$ into this description, which will result in us giving a more "precise" name for this object. Along the way, we will discover why $X$ had to be a finite complex and how to think about more general $X$. For now, though, we will content ourselves with investigating the Hopf algebra structure on $H \mathbb{F}_{2}^{*} \mathbb{R} \mathrm{P}^{\infty}$, the cohomology of an infinite complex.

Example 1.2.15 Recall that $\mathbb{R} \mathrm{P}^{\infty}$ is an $H$-space in two equivalent ways:

1. There is an identification $\mathbb{R P}^{\infty} \simeq K\left(\mathbb{F}_{2}, 1\right)$, and the $H$-space structure is induced by the sum on cohomology.

2. There is an identification $\mathbb{R} \mathrm{P}^{\infty} \simeq B O(1)$, and the $H$-space structure is induced by the tensor product of real line bundles.

In either case, this induces a Hopf algebra diagonal

$$
H \mathbb{F}_{2}^{*} \mathbb{R} P^{\infty} \otimes H \mathbb{F}_{2}^{*} \mathbb{R} P^{\infty} \stackrel{\Delta}{\leftarrow} H \mathbb{F}_{2}^{*} \mathbb{R} P^{\infty}
$$

$\overline{7}$ Additionally, the only values of $j$ for which $\mathbb{G}_{m}[j]$ is an infinitesimal thickening of $\mathbb{G}_{m}[1]$ are those of the form $j=2^{k}$. 
which we would like to analyze. This map is determined by where it sends the class $x$, and because it must respect gradings it must be of the form $\Delta x=a x_{1}+b x_{2}$ for some constants $a, b \in \mathbb{F}_{2}$. Furthermore, because it belongs to a Hopf algebra structure, it must satisfy the unitality axiom

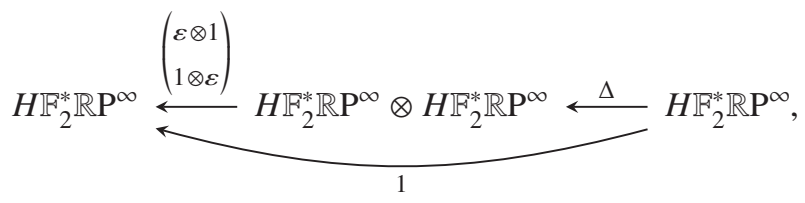

and hence it takes the form

$$
\Delta(x)=x_{1}+x_{2} .
$$

Noticing that this is exactly the diagonal map in Example 1.2.5, we tentatively identify " $\mathbb{R P}_{H \mathbb{F}_{2}}^{\infty}$ " with the additive group. This is extremely suggestive but does not take into account the fact that $\mathbb{R} \mathrm{P}^{\infty}$ is an infinite complex, so we have not yet allowed ourselves to write " $\mathbb{R P}_{H \mathbb{F}_{2}}^{\infty}$ " In light of the rest of the material discussed in this section, we have left open a very particular point: It is not clear if we should use the name " $\mathbb{G}_{a}$ " or " $\widehat{\mathbb{G}}_{a}$." We will straighten this out in the subsequent Lecture.

\subsection{The Steenrod Algebra}

We left off in the previous Lecture with an ominous finiteness condition in Definition 1.2.13, and we produced a pair of reasonable guesses as to what " $\mathbb{R P}_{H \mathbb{F}_{2}}^{\infty}$ " could mean in Example 1.2.15. We will decide which of the two guesses is correct by rigidifying the target category of Definition 1.2.13 so as to incorporate the following extra structures:

1. Cohomology rings are graded, and maps of spaces respect this grading.

2. Cohomology rings receive an action of the Steenrod algebra, and maps of spaces respect this action.

3. Both of these are made somewhat more complicated when taking the cohomology of an infinite complex.

4. (Cohomology rings for more elaborate cohomology theories are only skewcommutative, but "Spec" requires a commutative input.)

In this Lecture, we will address all these deficiencies of $X_{\mathrm{HF}_{2}}$ except for \#4, which does not matter with mod- 2 coefficients but which will be something of a bugbear throughout the rest of the book. 
We will begin by considering the grading on $H \mathbb{F}_{2}^{*} X$, where $X$ is a finite complex. In algebraic geometry, the following standard construction is used to track gradings: ${ }^{8}$

Definition 1.3.1 ([Str99b, definition 2.95]) $\quad \mathrm{A} \mathbb{Z}$-grading on a ring $A$ is a system of additive subgroups $A_{k}$ of $A$ satisfying $A=\bigoplus_{k} A_{k}, 1 \in A_{0}$, and $A_{j} A_{k} \subseteq A_{j+k}$. Additionally, a map $f: A \rightarrow S$ of graded rings is said to respect the grading if $f\left(A_{k}\right) \subseteq S_{k} .{ }^{9}$

Lemma 1.3.2 ([Str99b, proposition 2.96]) A graded ring $A$ is equivalent data to an affine scheme $\operatorname{Spec} A$ with an action by $\mathbb{G}_{m}$. Additionally, a map $A \rightarrow B$ is homogeneous exactly when the induced map $\operatorname{Spec} B \rightarrow \operatorname{Spec} B$ is $\mathbb{G}_{m}$-equivariant.

Proof A $\mathbb{G}_{m}$-action on $\operatorname{Spec} A$ is equivalent data to a coaction map

$$
\alpha^{*}: A \rightarrow A \otimes \mathbb{F}_{2}\left[x^{ \pm}\right] .
$$

Define $A_{k}$ to be those points in $a$ satisfying $\alpha^{*}(a)=a \otimes x^{k}$. It is clear that we have $1 \in A_{0}$ and that $A_{j} A_{k} \subseteq A_{j+k}$. To see that $A=\bigoplus_{k} A_{k}$, note that every tensor can be written as a sum of pure tensors. Conversely, given a graded ring $A$, define the coaction map on $A_{k}$ by

$$
\left(a_{k} \in A_{k}\right) \mapsto x^{k} a_{k}
$$

and extend linearly.

This notion from algebraic geometry is somewhat different from what we are used to in algebraic topology, essentially because the algebraic topologist's "cohomology ring" is not really a ring at all - one is only allowed to consider sums of homogeneous degree elements. This restriction stems directly from the provenance of cohomology rings: recall that

$$
H \mathbb{F}_{2}^{n} X:=\pi_{-n} F\left(\Sigma_{+}^{\infty} X, H \mathbb{F}_{2}\right) .
$$

One can only form sums internal to a particular homotopy group, using the cogroup structure on $\mathbb{S}^{-n}$. On the other hand, the most basic ring of algebraic geometry is the polynomial ring, and hence their definition is adapted to handle, for instance, the potential degree drop when taking the difference of two (nonhomogeneous) polynomials of the same degree.

${ }^{8}$ Strickland gives an alternative formalism for tracking gradings [Strb, sections 11 and 14] called a polarization, which amounts to choosing a trivialization $\pi_{2} E \cong \pi_{0} E$ and considering the isomorphisms $\pi_{2 n} E \cong\left(\pi_{2} E\right)^{\otimes_{\pi_{0}} E(n)}$.

9 The terminology "ZZ-filtering" might be more appropriate, but this is the language commonly used. 
We can modify our perspective very slightly to arrive at that of the algebraic geometers' by replacing $H \mathbb{F}_{2}$ with the periodified spectrum

$$
H \mathbb{F}_{2} P=\bigvee_{j=-\infty}^{\infty} \Sigma^{j} H \mathbb{F}_{2} .
$$

This spectrum forms a ring in the homotopy, where the ring multiplication is given by the following map for each summand of the smash product:

$$
\Sigma^{j} H \mathbb{F}_{2} \wedge \Sigma^{k} H \mathbb{F}_{2} \simeq \Sigma^{j+k}\left(H \mathbb{F}_{2} \wedge H \mathbb{F}_{2}\right) \stackrel{\Sigma^{j+k} \mu}{\longrightarrow} \Sigma^{j+k} H \mathbb{F}_{2} .
$$

This has the property that $H \mathbb{F}_{2} P^{0}(X)$ is isomorphic to $\bigoplus_{n} H \mathbb{F}_{2}^{n}(X)$ as ungraded rings, ${ }^{10}$ but now we can make topological sense of the sum of two classes that used to live in different $H \mathbb{F}_{2}$-degrees. At this point we can manually craft the desired coaction map $\alpha^{*}$ from Lemma 1.3.2, but we will shortly find that algebraic topology gifts us with it on its own.

Our route to finding this internally occurring $\alpha^{*}$ is by turning to the next supplementary structure: the action of the Steenrod algebra. Naively approached, this does not fit into the framework we have been sketching so far: The Steenrod algebra arises as the homotopy endomorphisms of $H \mathbb{F}_{2}$ and so is a noncommutative algebra. In turn, the action map

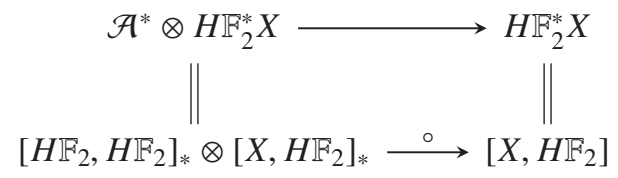

will be difficult to squeeze into any kind of algebro-geometric framework. Milnor was the first person to see a way around this, with two crucial observations. First, the Steenrod algebra is a Hopf algebra, ${ }^{11}$ using the map

$$
\left[H \mathbb{F}_{2}, H \mathbb{F}_{2}\right]_{*} \stackrel{\mu^{*}}{\rightarrow}\left[H \mathbb{F}_{2} \wedge H \mathbb{F}_{2}, H \mathbb{F}_{2}\right]_{*} \cong\left[H \mathbb{F}_{2}, H \mathbb{F}_{2}\right]_{*} \otimes\left[H \mathbb{F}_{2}, H \mathbb{F}_{2}\right]_{*}
$$

as the diagonal. This Hopf algebra structure is actually cocommutative - this is a rephrasing of the symmetry of the Cartan formula:

$$
\mathrm{Sq}^{n}(x y)=\sum_{i+j=n} \mathrm{Sq}^{i}(x) \mathrm{Sq}^{j}(y) .
$$

It follows that the linear-algebraic dual of the Steenrod algebra $\mathcal{A}_{*}$ is a commutative ring, and hence Spec $\mathcal{A}_{*}$ would make a reasonable algebro-geometric object.

10 This follows from the equivalence $\bigvee_{j=-\infty}^{\infty} \Sigma^{j} H \mathbb{F}_{2} \rightarrow \prod_{j=-\infty}^{\infty} \Sigma^{j} H \mathbb{F}_{2}$ and the finiteness of $X$.

11 The construction of both the Hopf algebra diagonal here and the coaction map that follows is somewhat ad hoc. We will give a more robust presentation in Lecture 3.1. 
Second, we want to identify the relationship between $\mathcal{A}_{*}$ and $H \mathbb{F}_{2}^{*} X$. Under the assumption that $X$ is a finite complex, the action map

$$
H \mathbb{F}_{2}^{*} X \leftarrow \mathcal{A}^{*} \otimes H \mathbb{F}_{2}^{*} X
$$

transposes under $\mathbb{F}_{2}$-linear duality to give a coaction map:

$$
\mathcal{A}_{*} \otimes H \mathbb{F}_{2}^{*} X \leftarrow H \mathbb{F}_{2}^{*} X .
$$

Finally, we pass to schemes to interpret this as an action map:

$$
\operatorname{Spec} \mathcal{A}_{*} \times X_{\mathrm{HF}_{2}} \stackrel{\alpha}{\rightarrow} X_{H \mathbb{F}_{2}} .
$$

Having produced the action map $\alpha$, we are now moved to study $\alpha$ as well as the structure group Spec $\mathcal{A}_{*}$ itself. Milnor works out the Hopf algebra structure of $\mathcal{A}_{*}$ by defining elements $\xi_{j} \in \mathcal{A}_{*}$ as follows. Taking $X=\mathbb{R} \mathrm{P}^{n}$ and $x \in H \mathbb{F}_{2}^{1}\left(\mathbb{R P}^{n}\right)$ the generator, he observes two things: first, $\mathrm{Sq}^{2^{j-1}} \cdots \mathrm{Sq}^{2^{0}} x=x^{2^{j}}$ is nonzero; and second, any other admissible sequence of squares applied to $x$ vanishes for degree reasons. It follows that the coaction applied to $x$ is supported exactly in cohomological degrees of the form $2^{j}$, which determines elements $\xi_{j}$ as in

$$
\lambda^{*}(x)=\sum_{j=0}^{\left\lfloor\log _{2} n\right\rfloor} x^{2^{j}} \otimes \xi_{j} \quad\left(\text { in } H \mathbb{F}_{2}^{*} \mathbb{R} \mathrm{P}^{n}\right) .
$$

Noticing that taking the limit $n \rightarrow \infty$ gives a well-defined infinite sum, he then makes the following calculation, stable in $n$ :

$$
\begin{aligned}
\left(\lambda^{*} \otimes 1\right) \circ \lambda^{*}(x) & =(1 \otimes \Delta) \circ \lambda^{*}(x), \\
\left(\lambda^{*} \otimes 1\right)\left(\sum_{j=0}^{\infty} x^{2^{j}} \otimes \xi_{j}\right) & \text { (coassociativity) } \\
& =\sum_{j=0}^{\infty}\left(\sum_{i=0}^{\infty} x^{2^{i}} \otimes \xi_{i}\right)^{2^{j}} \otimes \xi_{j} \quad \text { (ring homomorphism) } \\
& =\sum_{j=0}^{\infty}\left(\sum_{i=0}^{\infty} x^{2^{i+j}} \otimes \xi_{i}^{2^{j}}\right) \otimes \xi_{j}=(1 \otimes \Delta) \circ \lambda^{*}(x) .
\end{aligned}
$$

(characteristic 2)

Then, turning to the right-hand side:

$$
\begin{aligned}
& \sum_{j=0}^{\infty}\left(\sum_{i=0}^{\infty} x^{2^{i+j}} \otimes \xi_{i}^{2^{j}}\right) \otimes \xi_{j}=(1 \otimes \Delta)\left(\sum_{m=0}^{\infty} x^{2^{m}} \otimes \xi_{m}\right), \\
& \sum_{j=0}^{\infty}\left(\sum_{i=0}^{\infty} x^{2^{i+j}} \otimes \xi_{i}^{2^{j}}\right) \otimes \xi_{j}=\sum_{m=0}^{\infty} x^{2^{m}} \otimes \Delta\left(\xi_{m}\right),
\end{aligned}
$$


from which it follows that

$$
\Delta \xi_{m}=\sum_{i+j=m} \xi_{i}^{2^{j}} \otimes \xi_{j} .
$$

Finally, Milnor shows that this is the complete story:

Theorem 1.3.3 (Milnor [Mil58, theorem 2], [MT68, chapter 6], [Lura, proposition 13.1]) There is an isomorphism

$$
\mathcal{A}_{*} \cong \mathbb{F}_{2}\left[\xi_{1}, \xi_{2}, \ldots, \xi_{j}, \ldots\right]
$$

Flippant proof The definition of the elements $\xi_{j}$ determines a Hopf algebra map $\mathbb{F}_{2}\left[\xi_{1}, \xi_{2}, \ldots\right] \rightarrow \mathcal{A}_{*}$ and hence a dual map

$$
\mathcal{A}^{*} \rightarrow \mathbb{F}_{2}\left[\xi_{j} \mid j \geq 1\right]^{\vee} \text {. }
$$

This second map is injective: If it had a kernel, then this would produce a subalgebra of $\mathcal{A}^{*}$ that acts trivially on $H \mathbb{F}_{2}^{*} \mathbb{R P}^{\infty}$, but any nonconstant member of $\mathcal{A}^{*}$ acts nontrivially on $H \mathbb{F}_{2}^{*}\left(\mathbb{R P}^{\infty}\right)^{\times k}$ for $k \gg 0$, and hence nontrivially on $H \mathbb{F}_{2}^{*} \mathbb{R} P^{\infty}$ itself. Then, since $\mathcal{A}_{*}$ and the polynomial algebra are both of graded finite type, Milnor can conclude his argument by counting how many elements he has produced, comparing against how many Adem and Cartan found (which we will do ourselves in Lecture 4.1), and noting that he has exactly enough. ${ }^{12}$

We are now in a position to uncover the desired map $\alpha^{*}$ from earlier. In order to retell Milnor's story with $H \mathbb{F}_{2} P$ in place of $H \mathbb{F}_{2}$, note that there is a topological construction involving $H \mathbb{F}_{2}$ from which $\mathcal{A}_{*}$ emerges:

$$
\mathcal{A}_{*}:=\pi_{*}\left(H \mathbb{F}_{2} \wedge H \mathbb{F}_{2}\right) \text {. }
$$

Performing substitution on this formula gives the periodified dual Steenrod algebra:

$$
\mathcal{A} P_{0}:=\pi_{0}\left(H \mathbb{F}_{2} P \wedge H \mathbb{F}_{2} P\right)=H \mathbb{F}_{2} P_{0}\left(H \mathbb{F}_{2} P\right) \cong \mathcal{A}_{*}\left[\xi_{0}^{ \pm}\right]
$$

and taking the continuous linear dual of the action map

$$
H \mathbb{F}_{2} P^{0} X \leftarrow \mathcal{A} P^{0} \otimes H \mathbb{F}_{2} P^{0} X
$$

gives a coaction map as before, and hence an action map

$$
\operatorname{Spec} \mathcal{A} P_{0} \times X_{H \mathbb{F}_{2} P} \rightarrow X_{H \mathbb{F}_{2} P}
$$

12 The elements dual to $\xi_{j}$ can be explicitly identified as the Milnor primitives $Q_{j}$, defined by $Q_{1}=\mathrm{Sq}^{1}$ and by $Q_{j}=\left[Q_{j-1}, \mathrm{Sq}^{2^{j-1}}\right]$ for $j>1$ [Mil58, corollary 2]. 
Lemma 1.3.4 ([Goe08, formula 3.4, remark 3.14]) Projecting to the quotient Hopf algebra $\mathcal{A} P_{0} \rightarrow \mathbb{F}_{2}\left[\xi_{0}^{ \pm}\right]$gives exactly the $\mathbb{G}_{m}$-action map provided by Lemma 1.3.2.

Calculation The main topological observation is that there is an inclusion

$$
\begin{aligned}
\left(H \mathbb{F}_{2} \wedge H \mathbb{F}_{2}\right) P & = \\
\bigvee_{j} \Sigma^{j}\left(H \mathbb{F}_{2} \wedge H \mathbb{F}_{2}\right) & \subseteq \bigvee_{r, s} \Sigma^{r} H \mathbb{F}_{2} \wedge \Sigma^{s} H \mathbb{F}_{2} \\
& =H \mathbb{F}_{2} P \wedge H \mathbb{F}_{2} P
\end{aligned}
$$

which is compatible with the unit map as in

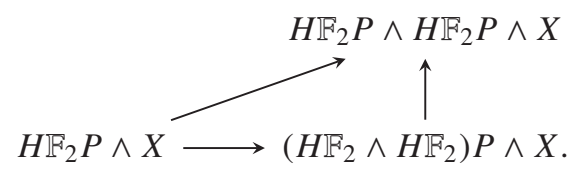

We now study how these inclusions interact with homogenization. Starting with an auxiliary cohomology class $x \in H \mathbb{F}_{2}^{n}(X)$, we produce a homogenized cohomology class $x \cdot u^{n} \in H \mathbb{F}_{2} P^{0}(X)$, and a class $\kappa \in \mathcal{A}_{n}$ similarly gives a homogenized class $\kappa u^{n} \in \mathcal{A} P_{0}$. The Steenrod coaction on $x$ admits expression as a sum

$$
\psi(x)=\sum_{I} x_{I} \prod_{j} \xi_{j}^{I_{j}}
$$

for classes $x_{I}$ with $\left|x_{I}\right|=|x|-\sum_{j}\left(2^{j}-1\right) I_{j}$. Under the periodified coaction map, the homogenized element $x u^{n}$ is sent to

$$
\psi\left(x u^{n}\right)=\sum_{I}\left(x_{I} u^{n-\sum_{j}\left(2^{j}-1\right) I_{j}}\right) \otimes \xi_{0}^{n-\sum_{j}\left(2^{j}-1\right) I_{j}} \prod_{j}\left(u^{2^{j}-1} \xi_{j}\right)^{I_{j}},
$$

where the invertible element $\xi_{0}=u^{-1} \otimes u \in \mathcal{A} P_{0}$ of degree 0 appears when shearing the powers of $u$ into the desired places in the formula. Projecting to the tensor factor of $\mathcal{A} P_{0}$ spanned by $\xi_{0}$, we thus compute

$$
\begin{gathered}
H \mathbb{F}_{2} P^{0}(X) \stackrel{\alpha^{*}}{\longrightarrow} H \mathbb{F}_{2} P^{0}(X) \otimes \mathcal{A} P_{0} \longrightarrow H \mathbb{F}_{2} P^{0}(X) \otimes \mathbb{F}_{2}\left[\xi_{0}^{ \pm}\right] \\
x \cdot u^{n} \longmapsto x \cdot u^{n} \otimes \xi_{0}^{n} .
\end{gathered}
$$

Applying Lemma 1.3.2 thus selects the original degree $n$ classes.

Early on in this discussion, trading the language "graded map" for " $\mathbb{G}_{m}$ equivariant map" did not seem to have much of an effect on our mathematics so little, in fact, that we will freely move between the graded homotopy groups 
of a spectrum and the zeroth homotopy group of a periodic spectrum throughout the rest of this book. ${ }^{13}$ The thrust of this lemma, however, is that "Steenrodequivariant map" already includes " $\mathbb{G}_{m}$-equivariant map," which is a visible gain in brevity. To study the rest of the content of Steenrod equivariance algebrogeometrically, we need only identify what the series $\lambda^{*}(x)$ embodies. Note that this necessarily involves some creativity, and the only justification we can supply will be moral, borne out over time, as our narrative encompasses more and more phenomena. With that caveat in mind, here is one such description. Recall the map induced by the $H$-space multiplication:

$$
H \mathbb{F}_{2} P^{0} \mathbb{R P}^{\infty} \otimes H \mathbb{F}_{2} P^{0} \mathbb{R} P^{\infty} \leftarrow H \mathbb{F}_{2} P^{0} \mathbb{R P}^{\infty} .
$$

Since this map comes from a map of spaces, it is equivariant for the Steenrod coaction, and since the action on the left is furthermore diagonal, we deduce the formula $\lambda^{*}\left(x_{1}+x_{2}\right)=\lambda^{*}\left(x_{1}\right)+\lambda^{*}\left(x_{2}\right)$.

Lemma 1.3.5 The series $\lambda(x)=\sum_{j=0}^{\infty} x^{2^{j}} \otimes \xi_{j}$ is the universal example of a series satisfying $\lambda\left(x_{1}+x_{2}\right)=\lambda\left(x_{1}\right)+\lambda\left(x_{2}\right)$. Points $f \in\left(\operatorname{Spec} \mathcal{A} P_{0}\right)(T)$ biject with those power series $\lambda_{f}$ with coefficients in the $\mathbb{F}_{2}$-algebra $T$ satisfying

$$
\lambda_{f}\left(x_{1}+x_{2}\right)=\lambda_{f}\left(x_{1}\right)+\lambda_{f}\left(x_{2}\right) \text {. }
$$

Proof Given a point $f \in\left(\operatorname{Spec} \mathcal{A} P_{0}\right)(T)$, we extract such a series by setting

$$
\lambda_{f}(x):=f^{*} \lambda(x)=\sum_{j=0}^{\infty} f\left(\xi_{j}\right) x^{2^{j}} \in T \llbracket x \rrbracket .
$$

Conversely, any series $\kappa(x)$ satisfying this homomorphism property must have nonzero terms appearing only in integer powers of 2 , and hence we can construct a point $f$ by declaring that $f$ sends $\xi_{j}$ to the $\left(2^{j}\right)$ th coefficient of $\kappa$.

We close our discussion by codifying what Milnor did when he stabilized against $n$. Each $\mathbb{R P}_{H \mathbb{F}_{2} P}^{n}$ is a finite affine scheme, and to make sense of the object $\mathbb{R P}_{H \mathbb{F}_{2} P}^{\infty}$ Milnor's technique was to consider the ind-system $\left\{\mathbb{R P}_{H \mathbb{F}_{2} P}^{n}\right\}_{n=0}^{\infty}$ of finite affine schemes. We will record this as our technique to handle general infinite complexes:

Definition 1.3.6 (see Definition 2.1.13) When $X$ is an infinite complex, filter it by its subskeleta $X^{(n)}$ and define $X_{H \mathbb{F}_{2} P}$ to be the ind-system $\left\{X_{H \mathbb{F}_{2} P}^{(n)}\right\}_{n=0}^{\infty}$ of finite schemes.

13 Generally speaking, certain computations are expressed most comfortably for an algebraic topologist in terms of graded objects, but we will endeavor to express the surrounding theory in the language of equivariance. 
This choice to follow Milnor resolves our uncertainty about the topological example from the previous lecture:

Example 1.3.7 (see Examples 1.2.11 and 1.2.15) Write $\widehat{\mathbb{G}}_{a}$ for the ind-system $\mathbb{A}^{1,(n)}$ with the group scheme structure given in Example 1.2.15. That this group scheme structure filters in this way is a simultaneous reflection of two facts:

1. Algebraic: The set $\widehat{\mathbb{G}}_{a}(T)$ consists of all nilpotent elements in $T$. The sum of two nilpotent elements of orders $n$ and $m$ is guaranteed to itself be nilpotent with order at most $n+m$.

2. Topological: There is a factorization of the multiplication map on $\mathbb{R} \mathrm{P}^{\infty}$ as $\mathbb{R} \mathrm{P}^{n} \times \mathbb{R} \mathrm{P}^{m} \rightarrow \mathbb{R} \mathrm{P}^{n+m}$ purely for dimensional reasons.

As group schemes, we have thus calculated

$$
\mathbb{R P}_{H \mathbb{F}_{2} P}^{\infty} \cong \widehat{\mathbb{G}}_{a}
$$

Example 1.3.8 Noting the homomorphism condition appearing in Lemma 1.3.5, we would like to connect $\operatorname{Spec} \mathcal{A} P_{0}$ with $\widehat{\mathbb{G}}_{a}$ more directly. Toward this end, we define a "hom functor" 14 for two formal schemes:

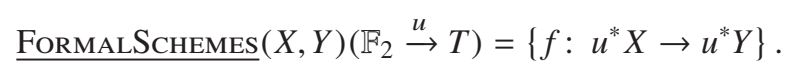

Restricting attention to homomorphisms, we see that a name for $\operatorname{Spec} \mathcal{A} P_{0}$ is

$$
\operatorname{Spec} \mathcal{A} P_{0} \cong \underline{\text { Aut }} \widehat{\mathbb{G}}_{a} \text {. }
$$

To check this, consider a point $g \in\left(\operatorname{Spec} \mathcal{A} P_{0}\right)(T)$ for an $\mathbb{F}_{2}$-algebra $T$. The $\mathbb{F}_{2}$-algebra structure of $T$ (which is uniquely determined by a property of $T$ ) give rise to a map $u: \operatorname{Spec} T \rightarrow \operatorname{Spec} \mathbb{F}_{2}$. The rest of the data of $g$ give rise to a power series in $T \llbracket x \rrbracket$ as in the proof of Lemma 1.3.5, which can be reinterpreted as an automorphism $g: u^{*} \widehat{\mathbb{G}}_{a} \rightarrow u^{*} \widehat{\mathbb{G}}_{a}$ of formal group schemes. ${ }^{15}$

Remark 1.3.9 The projection $\mathcal{A} P_{0} \rightarrow \mathbb{F}_{2}\left[\xi_{0}^{ \pm}\right]$is split as Hopf algebras, and hence there is a decomposition

$$
\underline{\text { Aut }} \widehat{\mathbb{G}}_{a} \cong \mathbb{G}_{m} \times \underline{\text { Aut }}_{1} \widehat{\mathbb{G}}_{a}
$$

where $\underline{\text { Aut }}_{1} \widehat{\mathbb{G}}_{a}$ consists of those automorphisms with leading coefficient $\xi_{0}$ exactly equal to 1 . This can be read to mean that the "interesting" part of the Steenrod algebra, $\underline{\text { Aut }}_{1} \widehat{\mathbb{G}}_{a}$, consists of stable operations, in the sense that their action is independent of the degree-tracking mechanism.

14 We are careful to say "functor" here because it is not generally another scheme.

15 This description, too, is sensitive to the difference between $\widehat{\mathbb{G}}_{a}$ and $\mathbb{G}_{a}$. The scheme End $\mathbb{G}_{a}$ is populated by polynomials satisfying a homomorphism condition, and essentially none of them have inverses. 
Example 1.3.10 Remembering the slogan

$$
\operatorname{Spec} \mathcal{A} P_{0} \cong \underline{\text { Aut }} \widehat{\mathbb{G}}_{a}
$$

also makes it easy to recall the structure formulas for the dual Steenrod algebra. For instance, consider the antipode map, which has the effect on Aut $\widehat{\mathbb{G}}_{a}$ of sending a power series to its compositional inverse. That is:

$$
\begin{aligned}
\sum_{j=0}^{\infty} \chi\left(\xi_{j}\right)\left(\sum_{k=0}^{\infty} \xi_{k} x^{2^{k}}\right)^{2^{j}} & =\sum_{j=0}^{\infty} \sum_{k=0}^{\infty} \chi\left(\xi_{j}\right) \xi_{k}^{2^{j}} x^{2^{j+k}} \\
& =\sum_{n=0}^{\infty}\left(\sum_{j+k=n} \chi\left(\xi_{j}\right) \xi_{k}^{2^{j}}\right) x^{2^{n}}=1,
\end{aligned}
$$

from which we can extract formulas like

$$
\chi\left(\xi_{0}\right)=\xi_{0}^{-1}, \quad \chi\left(\xi_{1}\right)=\xi_{0}^{-3} \xi_{1}, \quad \chi\left(\xi_{2}\right)=\xi_{0}^{-7} \xi_{1}^{3}+\xi_{0}^{-5} \xi_{2}, \quad \ldots
$$

Remark 1.3.11 Our interest in $\operatorname{colim}_{n} H \mathbb{F}_{2} P^{0} \mathbb{R} \mathrm{P}^{n}$ is the culmination of quite a lot of definitions in basic algebraic topology, quietly lurking in the background. For instance, infinite $\mathrm{CW}$-complexes are defined to have the weak topology coming from their skeleta, so that a continuous map off of such an infinite complex is continuous if and only if it is the colimit of a compatible system of maps. The cohomology of an infinite complex comes with a Milnor sequence, and if we arrange the situation so as not to encounter a $\lim ^{1}$ term, it is exactly equal to the limit of the cohomology groups of the finite stages - but because all maps between complexes filter through the finite stages, all the induced maps on cohomology are necessarily continuous for the adic topology. This is exactly the phenomenon we are capturing (or, indeed, enforcing in algebra) when we track the system of finite schemes $\left\{\mathbb{R P}_{H \mathbb{F}_{2} P}^{n}\right\}_{n=0}^{\infty}$.

In summary, the formula $\mathbb{R P}_{H \mathbb{F}_{2} P}^{\infty} \cong \widehat{\mathbb{G}}_{a}$ embodies the extremely good compression ratio of this approach: It simultaneously encodes the cohomology ring of $\mathbb{R} P^{\infty}$ as the formal scheme, its diagonal as the group scheme structure, and the coaction of the dual Steenrod algebra by the identification with Aut $\widehat{\mathbb{G}}_{a}$. As a separate wonder, it is also remarkable that the single cohomological calculation $\mathbb{R P}_{H \mathbb{F}_{2} P}^{\infty}$ exerts such enormous control over mod-2 cohomology itself (e.g., the entire structure of the dual Steenrod algebra). We will eventually give a concrete reason for this in Lecture 4.1, but it will also turn out to be a surprisingly common occurrence even in many situations in which such a direct link is not available. This is, already, one of the mysteries of the subject. 


\subsection{Hopf Algebra Cohomology}

In this section, we will focus on an important classical tool: the Adams spectral sequence. We are going to study this in greater earnest later on, so we will avoid giving a satisfying construction in this lecture. Still, even without a construction, it is instructive to see how this comes about from a moral perspective. Because we intend to use the Adams spectral sequence to perform computations, in this lecture we prefer to work with graded homology and cohomology.

Begin by considering the following three self-maps of the stable sphere:

$$
\mathbb{S}^{0} \stackrel{0}{\longrightarrow} \mathbb{S}^{0}, \quad \mathbb{S}^{0} \stackrel{1}{\longrightarrow} \mathbb{S}^{0}, \quad \mathbb{S}^{0} \stackrel{2}{\longrightarrow} \mathbb{S}^{0} .
$$

If we apply mod-2 homology to each line, the induced maps are

$$
\mathbb{F}_{2} \stackrel{0}{\longrightarrow} \mathbb{F}_{2}, \quad \mathbb{F}_{2} \stackrel{1}{\longrightarrow} \mathbb{F}_{2}, \quad \mathbb{F}_{2} \stackrel{0}{\longrightarrow} \mathbb{F}_{2} .
$$

We see that mod-2 homology can immediately distinguish between the null map and the identity map just by its behavior on morphisms, but it cannot distinguish between the null map and the multiplication-by-2 map. To try to distinguish between these two, we use the only other tool available to us: Homology theories send cofiber sequences to long exact sequences, and moreover the data of a map $f$ and the data of the inclusion map $\mathbb{S}^{0} \rightarrow C(f)$ into its cone are equivalent in the stable category. So, we trade our maps 0 and 2 for the following cofiber sequences:

$$
\mathbb{S}^{0} \longrightarrow C(0) \longrightarrow \mathbb{S}^{1}, \quad \mathbb{S}^{0} \longrightarrow C(2) \longrightarrow \mathbb{S}^{1} .
$$

The homology groups of these spectra $C(0)$ and $C(2)$ are more complicated than just that of $\mathbb{S}^{0}$, and we will draw them according to the following conventions: each "•" in the row labeled "[j]" indicates an $\mathbb{F}_{2}$-summand in the $j$ th $H \mathbb{F}_{2}$ homology of the spectrum. Applying homology to these cofiber sequences and drawing the results, these again appear to be identical:

[0]

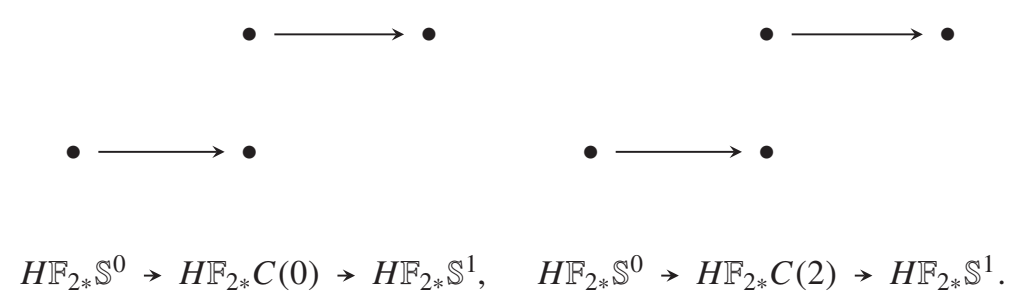

However, if we enrich our picture with the data we discussed in Lecture 1.3, we 
can finally see the difference. Recall the topological equivalences

$$
C(0) \simeq \mathbb{S}^{0} \vee \mathbb{S}^{1}, \quad C(2) \simeq \Sigma^{-1} \Sigma^{\infty} \mathbb{R} \mathrm{P}^{2}
$$

In the two cases, the coaction map $\lambda_{*}$ is given by

$$
\begin{array}{rlrl}
\lambda_{*}: H \mathbb{F}_{2 *} C(0) & \rightarrow H \mathbb{F}_{2 *} C(0) \otimes \mathcal{A}_{*} & \lambda_{*}: H \mathbb{F}_{2 *} C(2) & \rightarrow H \mathbb{F}_{2 *} C(2) \otimes \mathcal{A}_{*} \\
\lambda^{*}: e_{0} & \mapsto e_{0} \otimes 1 & \lambda^{*}: e_{0} & \mapsto e_{0} \otimes 1+e_{1} \otimes \xi_{1} \\
\lambda^{*}: e_{1} & \mapsto e_{1} \otimes 1, & \lambda^{*}: e_{1} \mapsto e_{1} \otimes 1 .
\end{array}
$$

We use a vertical line to indicate the nontrivial coaction involving $\xi_{1}$ :

[0]

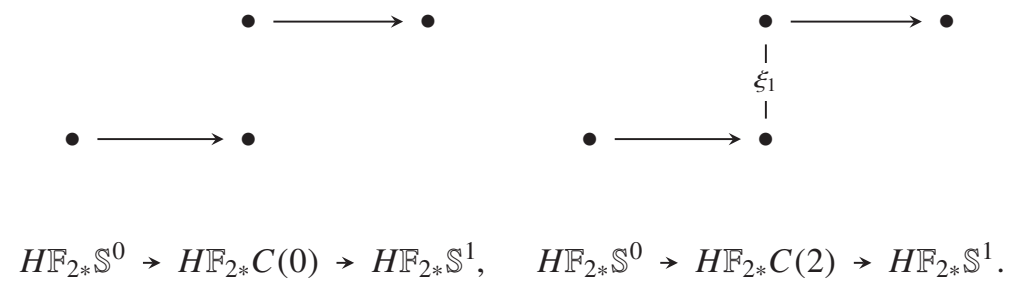

We can now see what trading maps for cofiber sequences has brought us: Mod-2 homology can distinguish the defining sequences for $C(0)$ and $C(2)$ by considering their induced extensions of comodules over $\mathcal{A}_{*} .{ }^{16}$ The Adams spectral sequence bundles this thought process into a single machine:

Theorem 1.4.1 ([Rav86, definition 2.1.8, lemma 2.1.16], [MT68, chapter 18]) The Adams spectral sequence is convergent and has signature

$$
\operatorname{Ext}_{\mathcal{A}_{*}^{* *}}^{*}\left(\mathbb{F}_{2}, \mathbb{F}_{2}\right) \Rightarrow\left(\pi_{*} \mathbb{S}^{0}\right)_{2}^{\wedge}
$$

In effect, this asserts that the above process is exhaustive: Every element of $\left(\pi_{*} \mathbb{S}^{0}\right)_{2}^{\wedge}$ can be detected and distinguished by some representative class of extensions of comodules for the dual Steenrod algebra. Mildly more generally, if $X$ is a bounded-below spectrum of finite type, then there is even an Adams spectral sequence of signature

$$
\operatorname{Ext}_{\mathcal{A}_{*}^{*}}^{*, *}\left(\mathbb{F}_{2}, H \mathbb{F}_{2 *} X\right) \Rightarrow \pi_{*} X_{2}^{\wedge} .
$$

We could now work through the construction of the Adams spectral sequence, but it will fit more nicely into a story later on in Lecture 3.1. Before moving on to other pursuits, however, we will record the following utility lemma. It is

16 More complicated homotopy elements may require more complicated extensions: For instance, the element $4=2 \cdot 2$ has to be written as a length- 2 extension to distinguish it from other elements. 
believable based on the above discussion, and we will need to use it before we get around to examining the guts of the construction.

Lemma 1.4.2 (see Remark 3.1.16) The 0-line of the Adams spectral sequence consists of exactly those elements visible to the Hurewicz homomorphism.

For the rest of this section, we will focus on an interpretation of the algebraic input "Ext $\mathcal{\mathscr { A }}_{*}^{* * *}\left(\mathbb{F}_{2}, H \mathbb{F}_{2 *} X\right)$," which will require us to grapple with the homological algebra of comodules for a Hopf algebra. To start that discussion, it's both reassuring and instructive to see that homological algebra can, in fact, even be done with comodules. In the usual development of homological algebra for modules, the key observations are the existence of projective and injective modules, and there is something similar at work here.

Remark 1.4.3 ([Rav86, appendix A1]) Much of the results that follow do not rely on working with a Hopf algebra over the field $k=\mathbb{F}_{2}$. In fact, $k$ can usually be taken to be a ring rather than a field. More generally, the theory goes through in the context of comodules over flat Hopf algebroids, see also Lemma 3.1.15.

Lemma 1.4.4 ([Rav86, definition A1.2.1]) Let A be a Hopf k-algebra, let $M$ be an A-comodule, and let $N$ be a k-module. There is a cofree adjunction:

$$
\operatorname{Comodules}_{A}\left(M, N \otimes_{k} A\right) \cong \operatorname{Modules}_{k}(M, N),
$$

where $N \otimes_{k} A$ is given the structure of an A-comodule by the coaction map

$$
N \otimes_{k} A \stackrel{1 \otimes \Delta}{\longrightarrow} N \otimes_{k}\left(A \otimes_{k} A\right)=\left(N \otimes_{k} A\right) \otimes_{k} A .
$$

Proof Given a map $f: M \rightarrow N$ of $k$-modules, we can build the composite

$$
M \stackrel{\psi_{M}}{\longrightarrow} M \otimes_{k} A \stackrel{f \otimes 1}{\longrightarrow} N \otimes_{k} A .
$$

Alternatively, given a map $g: M \rightarrow N \otimes_{k} A$ of $A$-comodules, we build the composite

$$
M \stackrel{g}{\rightarrow} N \otimes_{k} A \stackrel{1 \otimes \varepsilon}{\longrightarrow} N \otimes_{k} k=N .
$$

Corollary 1.4.5 ([Rav86, lemma A1.2.2]) The category Comodules $_{A}$ has enough injectives. Namely, if $M$ is an A-comodule and $M \rightarrow I$ is an inclusion of $k$-modules into an injective $k$-module $I$, then $M \rightarrow I \otimes_{k} A$ is an injective A-comodule under $M$.

Remark 1.4.6 In our case, $M$ itself is always $k$-injective, so there's already an injective map $\psi_{M}: M \rightarrow M \otimes A$ : the coaction map. The assertion that this map is coassociative is identical to saying that it is a map of comodules. 
Satisfied that "Ext" at least makes sense, we're free to pursue more conceptual ends. Recall from algebraic geometry that a module $M$ over a ring $A$ is equivalent data to quasicoherent sheaf $\widetilde{M}$ over Spec $A$. We now give a definition of "quasicoherent sheaf" that fits with our functorial perspective:

Definition 1.4.7 ([Hov02, definition 1.1], [Str99b, definition 2.42]) An assignment $\mathcal{F}: X(T) \rightarrow$ ModulEs $_{T}$ is said to be a presheaf (of modules) over $X$ when it satisfies lax functoriality in $T$ : There is a natural transformation $\tau$ such that for each map $f: T \rightarrow T^{\prime}, \tau$ describes a map (between the not necessarily equal composites)

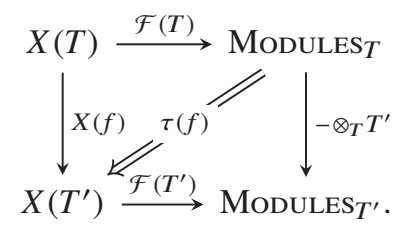

We think of the image of a particular point $t: \operatorname{Spec} T \rightarrow X$ in $\operatorname{Modules}_{T}$ as the module of "sections over $t$." Such a presheaf is said to be a quasicoherent sheaf when these natural transformations are all natural isomorphisms.

Lemma 1.4.8 ([Str99b, proposition 2.47]) An A-module $M$ gives rise to a quasicoherent sheaf $\widetilde{M}$ on Spec $A$ by the rule

$$
(\operatorname{Spec} T \rightarrow \operatorname{Spec} A) \mapsto M \otimes_{A} T \text {. }
$$

Conversely, every quasicoherent sheaf over an affine scheme arises in this way.

The tensoring operation appearing in the definition of a presheaf appears more generally as an operation on the category of sheaves.

Definition 1.4.9 A map $f: \operatorname{Spec} B \rightarrow \operatorname{Spec} A$ induces maps $f^{*} \dashv f_{*}$ of categories of quasicoherent sheaves. At the level of modules, these are given by

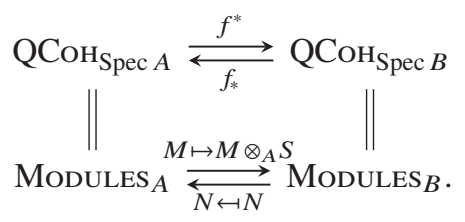

One of the main uses of these operations is to define the cohomology of a sheaf. Let $\pi: X \rightarrow$ Spec $k$ be a scheme over Spec $k, k$ a field, and let $\mathcal{F}$ be a sheaf over $X$. The adjunction above induces a derived adjunction

$$
\operatorname{Ext}_{X}\left(\pi^{*} k, \mathcal{F}\right) \cong \operatorname{Ext}_{\operatorname{Spec} k}\left(k, R \pi_{*} \mathcal{F}\right),
$$


which is used to translate the definition of sheaf cohomology to that of the cohomology of the derived pushforward $R \pi_{*} \mathcal{F}$, itself interpretable as a mere complex of $k$-modules. This pattern is very general: The sense of "cohomology" relevant to a situation is often accessed by taking the derived pushforward to a suitably terminal object. ${ }^{17}$ To invent a notion of cohomology for comodules over a Hopf algebra, we are thus moved to produce push and pull functors for a map of Hopf algebras, and this is best motivated by another example.

Example 1.4.10 A common source of Hopf algebras is through group-rings: Given a group $G$, we can define the Hopf $k$-algebra $k[G]$ consisting of formal $k$-linear combinations of elements of $G$. This Hopf algebra is commutative exactly when $G$ is abelian, and $k[G]$-modules are naturally equivalent to $k$ linear $G$-representations. Dually, the ring $k^{G}$ of $k$-valued functions on $G$ is always commutative, using pointwise multiplication of functions, and it is cocommutative exactly when $G$ is abelian. If $G$ is finite, then $k^{G}$ and $k[G]$ are $k$-linear dual Hopf algebras, and hence finite-dimensional $k^{G}$-comodules are naturally equivalent to finite-dimensional $k$-linear $G$-representations. ${ }^{18}$

A map of groups $f: G \rightarrow H$ induces a map $k^{f}: k^{H} \rightarrow k^{G}$ of Hopf algebras, and it is reasonable to expect that the induced push and pull maps of comodules mimic those of $G$ - and $H$-representations. Namely, given an $H$-representation $M$, we can produce a corresponding $G$-representation by precomposition with $f$. However, given a $G$-representation $N$, two features may have to be corrected to extract an $H$-representation:

1. If $f$ is not surjective, we must decide what to do with the extra elements in $H$.

2. If $f$ is not injective - say, $f\left(g_{1}\right)=f\left(g_{2}\right)$ - then we must force the behavior of the extracted $H$-representation to agree on $f\left(g_{1}\right)$ and $f\left(g_{2}\right)$, even if $g_{1}$ and $g_{2}$ act differently on $N$. In the extreme case of $f: G \rightarrow 1$, we expect to recover the fixed points of $N$, since this pushforward computes $H_{\mathrm{gp}}^{0}(G ; N)$.

These concerns, together with the definition of a tensor product as a coequalizer, motivate the following:

17 This perspective often falls under the heading of "six-functor formalism."

18 There is a variation on this equivalence that uses fewer dualities and which is instructive to expand. The Hopf algebra $k^{G}=\prod_{g \in G} k$ is the ring of functions on the constant group scheme $G$, and its $k$-points $\left(\operatorname{Spec} k^{G}\right)(k)$ biject with points in $G$. Namely, given $g \in G$ we can form a projection map $g: k^{G} \rightarrow k$ and hence a composite $M \rightarrow M \otimes_{k} k^{G} \stackrel{1 \otimes g}{\longrightarrow} M \otimes_{k} k \cong M$. Collectively, this determines a map $G \times M \rightarrow M$ witnessing $M$ as a $G$-representation. In the other direction, if $G$ is finite then we can construct a map $M \rightarrow M \otimes_{k} \prod_{g \in G} k$ sending $m \in M$ to $g \cdot m$ in the $g$ th labeled component of the target. 
Definition 1.4.11 Given $A$-comodules $M$ and $N$, their cotensor product is the $k$-module defined by the equalizer

$$
M \square_{A} N \rightarrow M \otimes_{k} N \stackrel{\psi_{M} \otimes 1-1 \otimes \psi_{N}}{\longrightarrow} M \otimes_{k} A \otimes_{k} N .
$$

Lemma 1.4.12 Given a map $f: A \rightarrow B$ of Hopf k-algebras, the induced adjunction $f^{*}+f_{*}$ is given at the level of comodules by

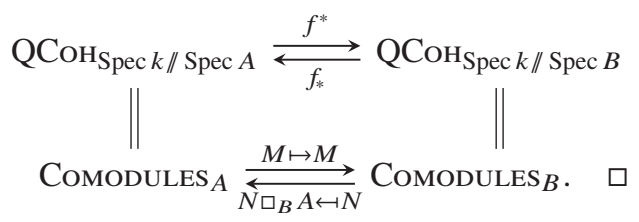

Remark 1.4.13 In Lecture 3.1 (and Definition 3.1.13 specifically), we will explain the notation "Spec $k / / \operatorname{Spec} A$ " used above. For now, suffice it to say that there again exists a functor-of-points notion of "quasicoherent sheaf" associated to a Hopf $k$-algebra $A$, and such sheaves are equivalent to $A$-comodules.

As an example application, cotensoring gives rise to a concise description of what it means to be a comodule map:

Lemma 1.4.14 ([Rav86, lemma A1.1.6b]) Let $M$ and $N$ be A-comodules with $M$ projective as a $k$-module. Then there is an equivalence

$$
\operatorname{Comodules}_{A}(M, N)=\operatorname{Modules}_{k}(M, k) \square_{A} N .
$$

This has two pleasant consequences. The first is that we can deduce a connection between the push-pull flavor of comodule cohomology described above and the input to the Adams spectral sequence:

Corollary 1.4.15 There is an isomorphism

$$
\operatorname{Comodules}_{A}(k, N)=\operatorname{Modules}_{k}(k, k) \square_{A} N=k \square_{A} N
$$

and hence

$$
\operatorname{Ext}_{A}(k, N) \cong \operatorname{Cotor}_{A}(k, N)\left(=H^{*} R \pi_{*} N\right) .
$$

Proof Resolve $N$ using the cofree modules described above, then apply either functor $\operatorname{ComodulEs}_{A}(k,-)$ or $k \square_{A}-$. In both cases, you get the same complex.

The second is that cofree comodules are cotorsion-free:

Corollary 1.4.16 Let $N=N^{\prime} \otimes_{k}$ A be a cofree comodule. Then $N \square_{A} k=N^{\prime}$. 
Proof Picking $M=k$, we have

$$
\begin{aligned}
\operatorname{Modules}_{k}\left(k, N^{\prime}\right) & =\operatorname{Comodules}_{A}(k, N) \\
& =\operatorname{Modules}_{k}(k, k) \square_{A} N \\
& =k \square_{A} N .
\end{aligned}
$$

Example 1.4.17 In Lecture 1.3, we identified $\mathcal{A}_{*}$ with the ring of functions on the group scheme $\underline{A u t}_{1}\left(\widehat{\mathbb{G}}_{a}\right)$ of strict automorphisms of $\widehat{\mathbb{G}}_{a}$, which is defined by the kernel sequence

$$
0 \rightarrow \underline{\text { Aut }}_{1}\left(\widehat{\mathbb{G}}_{a}\right) \rightarrow \underline{\text { Aut }}\left(\widehat{\mathbb{G}}_{a}\right) \rightarrow \mathbb{G}_{m} \rightarrow 0 .
$$

The punchline is that this is analogous to Example 1.4.10: The object $\operatorname{Cotor}_{\mathcal{A}_{*}}\left(\mathbb{F}_{2}, H \mathbb{F}_{2 *} X\right)$ is thought of as the "derived fixed points" of $\underline{\text { Aut }}_{1}\left(\widehat{\mathbb{G}}_{a}\right)$ acting on $H \mathbb{F}_{2 *} X$.

We now give several examples to get a sense of how the Adams spectral sequence behaves.

Example 1.4.18 Consider the degenerate case $X=H \mathbb{F}_{2}$. Then $H \mathbb{F}_{2 *}\left(H \mathbb{F}_{2}\right)=$ $\mathcal{A}_{*}$ is a cofree comodule, and hence Cotor is concentrated on the 0-line:

$$
\operatorname{Cotor}_{\mathcal{A}_{*}}^{* *}\left(\mathbb{F}_{2}, H \mathbb{F}_{2 *}\left(H \mathbb{F}_{2}\right)\right)=\mathbb{F}_{2} \text {. }
$$

The Adams spectral sequence collapses to show the wholly unsurprising equality $\pi_{*} H \mathbb{F}_{2}=\mathbb{F}_{2}$, and indeed this is the element in the image of the Hurewicz map $\pi_{*} H \mathbb{F}_{2} \rightarrow H \mathbb{F}_{2 *} H \mathbb{F}_{2}$.

Example 1.4.19 In the slightly less degenerate case of $X=H \mathbb{Z}$, one can calculate

$$
\operatorname{Cotor}_{\mathcal{A}_{*}^{*}}^{* *}\left(\mathbb{F}_{2}, H \mathbb{F}_{2 *} H \mathbb{Z}\right) \cong \operatorname{Cotor}_{\Lambda\left[\xi_{1}\right]}^{*, *}\left(\mathbb{F}_{2}, \mathbb{F}_{2}\right) \cong \mathbb{F}_{2}\left[h_{0}\right] .
$$

This spectral sequence collapses, and the additive extensions cause it to converge to $\mathbb{Z}_{2}^{\wedge}$ in degree 0 and 0 in all other degrees. The governing element $\xi_{1}$ is precisely the 2-adic Bockstein, which mediates the difference between trivial and nontrivial extensions of $\mathbb{Z} / 2^{j}$ by $\mathbb{Z} / 2$.

Example 1.4.20 Next, we consider the more computationally serious case of $X=k O$, the connective real $K$-theory spectrum. The main input we need is the structure of $\mathrm{HF}_{2 *} k O$ as an $\mathcal{A}_{*}$-comodule, so that we can compute

$$
\operatorname{Cotor}_{\mathcal{A}_{*}}^{* * *}\left(\mathbb{F}_{2}, H \mathbb{F}_{2 *} k O\right) \Rightarrow \pi_{*} k O_{2}^{\wedge} \text {. }
$$

There is a slick trick for doing this: By working in the category of $\mathrm{kO}$-modules rather than in all spectra, we can construct a relative Adams spectral sequence

$$
\operatorname{Cotor}_{\pi_{*} H \mathbb{F}_{2} \wedge_{k} O H \mathbb{F}_{2}}^{* *}\left(\mathbb{F}_{2}, \pi_{*} H \mathbb{F}_{2} \wedge_{k} O\left(k O \wedge H \mathbb{F}_{2}\right)\right) \Rightarrow \pi_{*}\left(k O \wedge H \mathbb{F}_{2}\right) .
$$


The second argument is easy to identify:

$$
\pi_{*} H \mathbb{F}_{2} \wedge_{k} O\left(k O \wedge H \mathbb{F}_{2}\right)=\pi_{*} H \mathbb{F}_{2} \wedge H \mathbb{F}_{2}=\mathcal{A}_{*} .
$$

The Hopf algebra requires further input. Consider the following trio of cofiber sequences: ${ }^{19}$

$$
\Sigma k O \stackrel{\cdot \eta}{\longrightarrow} k O \rightarrow k U, \quad \Sigma^{2} k U \stackrel{\cdot \beta}{\rightarrow} k U \rightarrow H \mathbb{Z}, \quad H \mathbb{Z} \stackrel{\cdot 2}{\rightarrow} H \mathbb{Z} \rightarrow H \mathbb{F}_{2} .
$$

These combine to give a resolution of $\mathrm{HF}_{2}$ via an iterated cofiber of free $k O$-modules, with Poincaré series

$$
\left(\left(1+t^{2}\right)+t^{3}\left(1+t^{2}\right)\right)+t\left(\left(1+t^{2}\right)+t^{3}\left(1+t^{2}\right)\right)=1+t+t^{2}+2 t^{3}+t^{4}+t^{5}+t^{6} .
$$

Repeatedly using the identity $k O \wedge_{k} O H \mathbb{F}_{2} \simeq H \mathbb{F}_{2}$ gives a small presentation of the Hopf algebra $\pi_{*} H \mathbb{F}_{2} \wedge_{k} O H \mathbb{F}_{2}$ : It is a commutative Hopf algebra over $\mathbb{F}_{2}$ with the above Poincaré series. The Borel-Milnor-Moore classification of commutative Hopf algebras over $\mathbb{F}_{2}[\mathrm{MM} 65$, theorem 7.11] shows that the algebra structure is either

$$
\frac{\mathbb{F}_{2}[a, b, c]}{\left(a^{2}=0, b^{2}=0, c^{2}=0\right)} \quad \text { or } \quad \frac{\mathbb{F}_{2}[a, b, c]}{\left(a^{2}=b, b^{2}=0, c^{2}=0\right)}
$$

for $|a|=1,|b|=2$, and $|c|=3$. By knowing that the natural map $\mathcal{A} \rightarrow$ $\pi_{*} H \mathbb{F}_{2} \wedge_{k} O H \mathbb{F}_{2}$ winds up inducing an isomorphism $\pi_{* \leq 2} \mathbb{S} \rightarrow \pi_{* \leq 2} k O$, we conclude that we are in the latter case, which gives a presentation of the Hopf algebra as a whole:

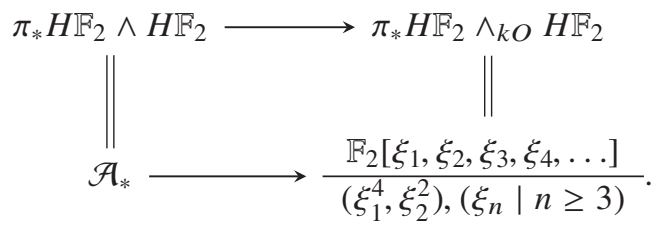

This Hopf algebra is commonly denoted $\mathcal{A}(1)_{*}$, and its corresponding subgroup scheme Spec $\pi_{*} H \mathbb{F}_{2} \wedge_{k} O H \mathbb{F}_{2} \subseteq \underline{\text { Aut }}_{1} \widehat{\mathbb{G}}_{a}$ allows easy memorization: It is the subscheme of automorphisms of the form $x+\xi_{1} x^{2}+\xi_{2} x^{4}$, with exactly the additional relations imposed on $\xi_{1}$ and $\xi_{2}$ so that this set is stable under composition and inversion. ${ }^{20,21}$

19 This first sequence, known as the Wood cofiber sequence, is a consequence of a very simple form of Bott periodicity [Har80, section 5]: There is a fiber sequence of infinite-loopspaces $O / U \rightarrow B O \rightarrow B U$, and $\underline{k O}_{1}=O / U$.

20 A similar analysis shows that $\pi_{*}\left(H \mathbb{F}_{2} \wedge_{H \mathbb{Z}} H \mathbb{F}_{2}\right)$ corepresents the subscheme of automorphisms of the form $x+\xi_{1} x^{2}$, which are stable under composition and inversion.

21 There is also an accidental isomorphism of this Hopf algebra with $\mathbb{F}_{2}^{D_{4}}$, where $D_{4}$ is the dihedral group with eight elements. 
Formal geometry aside, this $k O$-based Adams spectral sequence collapses, giving an isomorphism

$$
\operatorname{Cotor}_{\mathcal{A}(1)_{*}^{*}}^{* *}\left(\mathbb{F}_{2}, \mathcal{A}_{*}\right)=\mathcal{A}_{*} / / \mathcal{A}(1)_{*} \cong H \mathbb{F}_{2 *} k O \text {. }
$$

In turn, the original Adams spectral sequence takes the form

$$
\operatorname{Cotor}_{\mathcal{A}_{*}}^{*, *}\left(\mathbb{F}_{2}, \mathcal{A} / / \mathcal{A}(1)_{*}\right) \cong \operatorname{Cotor}_{\mathcal{A}(1)_{*}}^{*, *}\left(\mathbb{F}_{2}, \mathbb{F}_{2}\right) \Rightarrow \pi_{*} k O
$$

where we have used the cofreeness property of Corollary 1.4.16. This spectral sequence is also collapsing, and we provide a picture of it in Figure 1.1. In particular, eight-fold real Bott periodicity can be quickly read off from this picture.

Example 1.4.21 At the other extreme, we can pick the extremely nondegenerate case $X=\mathbb{S}$, where $\underline{\text { Aut }}_{1} \widehat{\mathbb{G}}_{a}$ acts maximally nonfreely on $\widetilde{\mathbb{F}}_{2}$. The resulting spectral sequence is pictured through a range in Figure 1.2. It is worth remarking that some of the stable stems receive identifiable names in this language: for instance, the elements of $H_{\mathrm{gp}}^{1}\left(\underline{\mathrm{Aut}}_{1}\left(\widehat{\mathbb{G}}_{a}\right) ; \widetilde{\mathbb{F}}_{2}\right)$ are exactly the 1 -cocycles

$$
h_{j}:\left(f=x+\sum_{j=1}^{\infty} \xi_{j} x^{2^{j}}\right) \mapsto \xi_{j} .
$$

The cocycle $h_{j}$ transforms in the $\mathbb{G}_{m}$-character $z \mapsto z^{2^{j}}$, hence $h_{1}$ is a name for the lone element in the spectral sequence contributing to $\pi_{1} \mathbb{S}$, which we also know to be $\eta$. In general, the elements $h_{j}$ selecting the power series coefficients are called the Hopf invariant 1 elements, and their survival or demise in the Adams spectral sequence is directly related to the problem of putting $H$-space structures on spheres [Ada62].

\subsection{The Unoriented Bordism Ring}

Our goal in this section is to use our results so far to make a calculation of $\pi_{*} M O$, the unoriented bordism ring. Our approach is the same as in the examples at the end of the previous section: We will want to use the Adams spectral sequence of signature

$$
\left.H_{\mathrm{gp}}^{*} \underline{\text { Aut }}_{1}\left(\widehat{\mathbb{G}}_{a}\right) ; H \mathbb{F}_{2} P_{0}(M O)\right) \Rightarrow \pi_{*} M O,
$$

where $H \mathbb{F}_{2} P_{0}(M O)$ denotes the associated quasicoherent sheaf over Spec $k / /$ Spec $A_{*}$ as in Remark 1.4.13 - and we will therefore want to understand $H \mathbb{F}_{2} P_{0}(M O)$ as a comodule for the dual Steenrod algebra. 


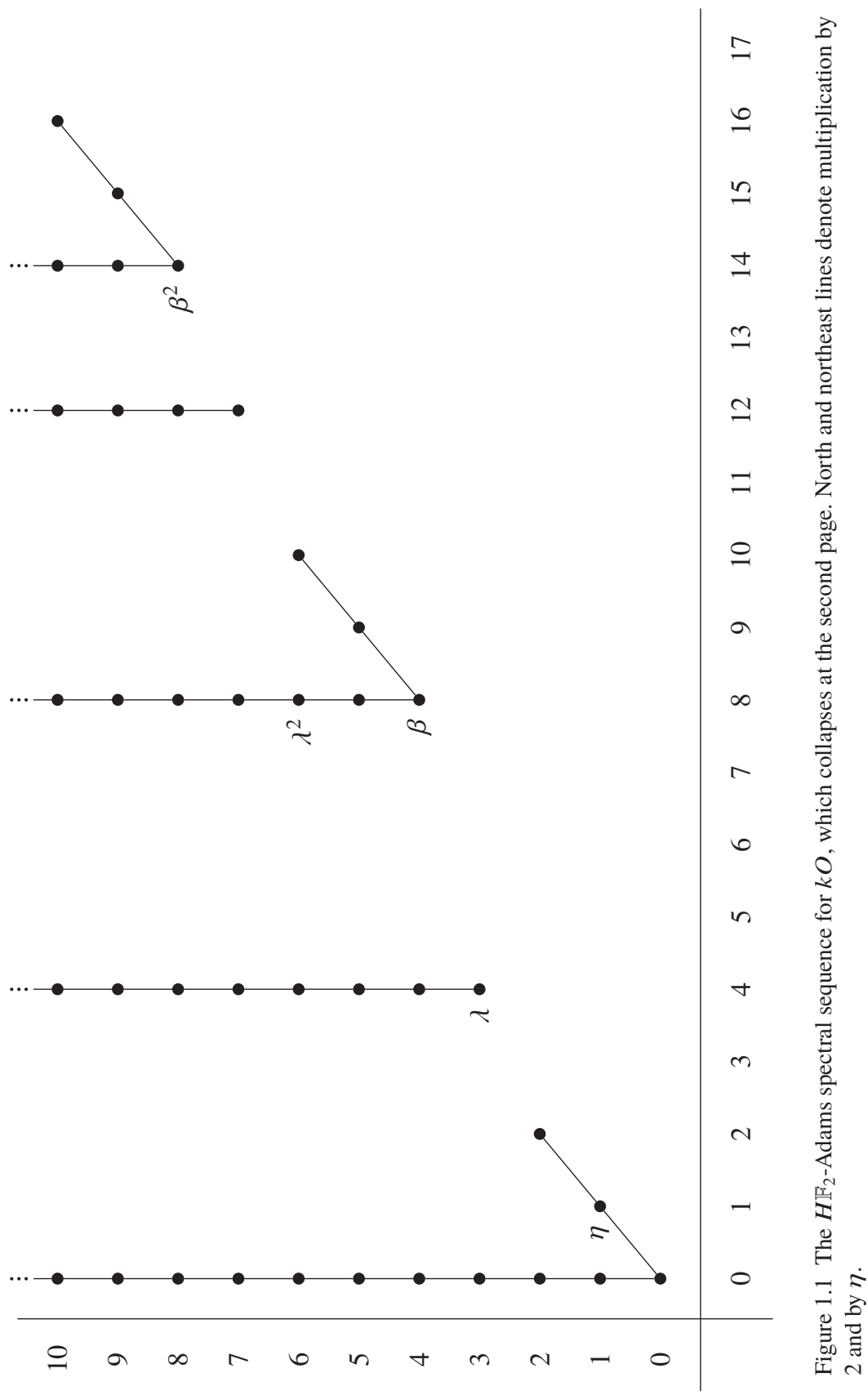




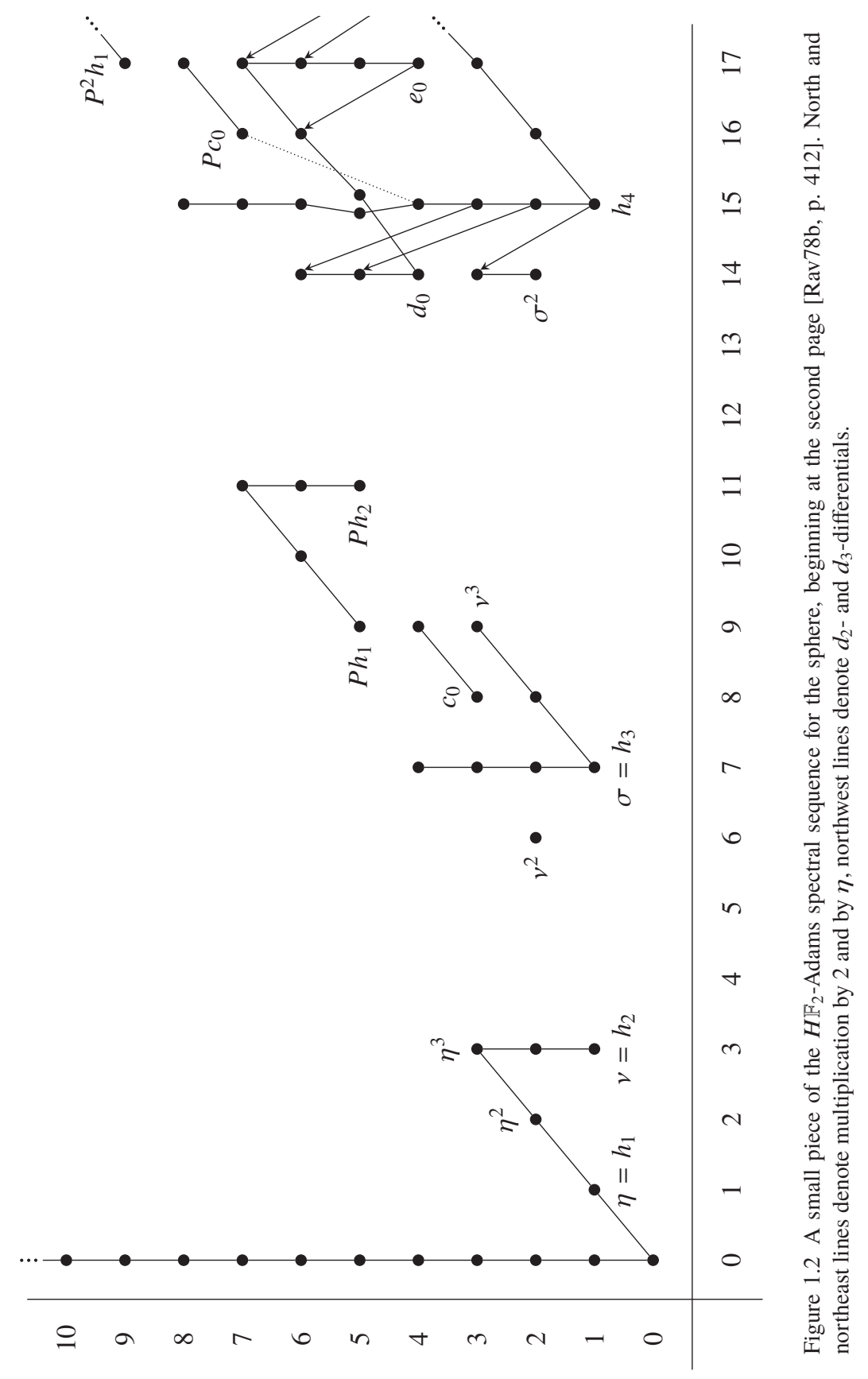


Our first step toward this is the following calculation:

Lemma 1.5.1 ([Swi02, theorem 16.17]) The natural map

$$
\widetilde{H \mathbb{F}}_{2} P_{0}(B O(1)) \rightarrow H \mathbb{F}_{2} P_{0}(B O)
$$

induces a map

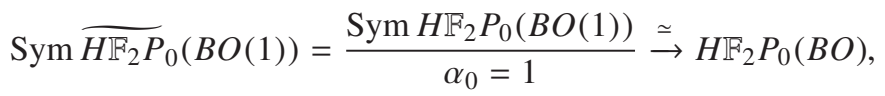

which is an isomorphism of Hopf algebras and of comodules for the dual Steenrod algebra.

Proof This follows from several standard facts about Stiefel-Whitney classes. First, these classes generate the cohomology ring $H \mathbb{F}_{2} P^{0} B O(n)$ [Swi02, theorem 16.10]:

$$
H \mathbb{F}_{2} P^{0} B O(n) \cong \mathbb{F}_{2} \llbracket w_{1}, \ldots, w_{n} \rrbracket .
$$

Then, the total Stiefel-Whitney class $w(V)=\sum_{j} w_{j}(V) t^{j}$ is exponential [Swi02, theorem 16.3], in the sense that

$$
w(V \oplus W)=w(V) \cdot w(W) .
$$

It follows that the natural map

$$
H \mathbb{F}_{2} P^{0} B O(n) \stackrel{\bigoplus_{j=1}^{n} \mathcal{L}_{j}}{\longrightarrow} H \mathbb{F}_{2} P^{0} B O(1)^{\times n} \cong\left(H \mathbb{F}_{2} P^{0} B O(1)\right)^{\otimes n}
$$

is the inclusion of the symmetric polynomials, by calculating the total StiefelWhitney class:

$$
w\left(\bigoplus_{j=1}^{n} \mathcal{L}_{j}\right)=\prod_{j=1}^{n}\left(1+w_{1}\left(\mathcal{L}_{j}\right)\right)=\sum_{j=0}^{n} \sigma_{j}\left(w_{1}\left(\mathcal{L}_{1}\right), \ldots, w_{1}\left(\mathcal{L}_{n}\right)\right) t^{j} .
$$

Dually, the homological map

$$
\left(H \mathbb{F}_{2} P_{0} B O(1)\right)^{\otimes n} \rightarrow H \mathbb{F}_{2} P_{0} B O(n)
$$

is surjective, modeling the quotient from the tensor product to the symmetric tensor product. Stabilizing as $n \rightarrow \infty$, we recover the statement of the lemma.

With this in hand, we now turn to the homotopy ring $\mathrm{HF}_{2} \mathrm{P}_{0} M O$. There are two equivalences that we might consider employing. We have the Thom isomorphism:

$$
\begin{gathered}
H \mathbb{F}_{2} P_{0}(B O(1))=H \mathbb{F}_{2} P_{0}(M O(1)) \\
\alpha_{j}, j \geq 0 \longmapsto \alpha_{j}^{\prime}, j \geq 0,
\end{gathered}
$$


and we also have the equivalence induced by the topological map in Example 1.1.3:

$$
\begin{gathered}
{\widetilde{H \mathbb{F}_{2} P_{0}}}(B O(1))=H \mathbb{F}_{2} P_{0}(\Sigma M O(1)) \\
\alpha_{j}, j \geq 1 \longmapsto \alpha_{j-1}^{\prime}, j \geq 1 .
\end{gathered}
$$

We will use them both in turn.

Corollary 1.5.2 ([Ada95, section I.3], [Hop, proposition 6.2]) There is again an isomorphism

$$
H \mathbb{F}_{2} P_{0}(M O) \cong \frac{\operatorname{Sym} H \mathbb{F}_{2} P_{0} M O(1)}{b_{0}^{\prime}=1}
$$

of rings and of comodules for the dual Steenrod algebra.

Proof The block sum maps

$$
B O(n) \times B O(m) \rightarrow B O(n+m)
$$

Thomify to give compatible maps

$$
M O(n) \wedge M O(m) \rightarrow M O(n+m) .
$$

Taking the colimit, this gives a ring structure on $M O$ compatible with that on $\Sigma_{+}^{\infty} B O$ and compatible with the Thom isomorphism.

We now seek to understand the utility of the scheme $\operatorname{Spec} H \mathbb{F}_{2} P_{0}(M O)$, as well as its action of $\underline{\operatorname{Aut}}\left(\widehat{\mathbb{G}}_{a}\right)$. The first of these tasks comes from untangling some of the topological dualities we've been using thus far.

Lemma 1.5.3 The following square commutes:

$$
\begin{aligned}
& \operatorname{Modules} \mathbb{F}_{2}\left(H \mathbb{F}_{2} P_{0}(M O), \mathbb{F}_{2}\right)=\operatorname{Spectra}\left(M O, H \mathbb{F}_{2} P\right) \\
& \uparrow \\
& \operatorname{Algebras}_{\mathbb{F}_{2} /}\left(H \mathbb{F}_{2} P_{0}(M O), \mathbb{F}_{2}\right)=\operatorname{Ring} \operatorname{Spectra}\left(M O, H \mathbb{F}_{2} P\right) \text {. }
\end{aligned}
$$

Proof The top isomorphism asserts that $\mathbb{F}_{2}$-cohomology and $\mathbb{F}_{2}$-homology are linearly dual to one another. The second follows immediately from investigating the effect of the ring homomorphism diagrams in the bottom-right corner in terms of the subset they select in the top-left.

Corollary 1.5.4 There is a bijection between homotopy classes of ring maps $\mathrm{MO} \rightarrow \mathrm{HF}_{2} \mathrm{P}$ and homotopy classes of factorizations

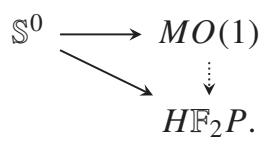


Proof We extend the square in Lemma 1.5.3 using the following diagram:

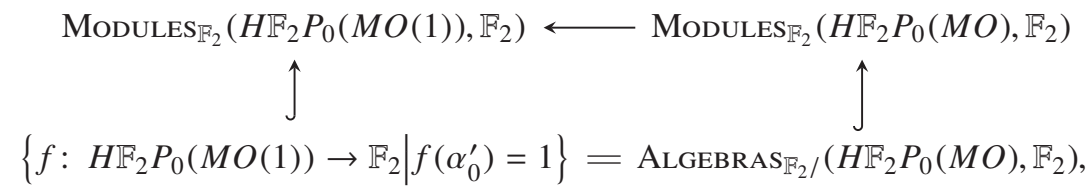

where the equality at bottom follows from the universal property of $H \mathbb{F}_{2} P_{0}(M O)$ in $\mathbb{F}_{2}$-algebras expressed in Corollary 1.5.2. Noting that $\alpha_{0}^{\prime}$ is induced by the topological map $\mathbb{S}^{0} \rightarrow M O(1)$, the condition $f\left(\alpha_{0}^{\prime}\right)=1$ is exactly the condition expressed in the statement of the corollary.

Corollary $\mathbf{1 . 5 . 5}$ ([Str06, definition 12.1, theorem 12.3]) There is an $\underline{\text { Aut }}\left(\widehat{\mathbb{G}}_{a}\right)$ equivariant isomorphism of schemes

$$
\operatorname{Spec} H \mathbb{F}_{2} P_{0}(M O) \cong \operatorname{Coord}_{1}\left(\mathbb{R P}_{H \mathbb{F}_{2} P}^{\infty}\right)
$$

where the latter is the subscheme of functions $\mathbb{R P}_{H \mathbb{F}_{2} P}^{\infty} \rightarrow \widehat{\mathbb{A}}^{1}$ which are coordinates (i.e., which are isomorphisms of formal schemes - or, equivalently, which restrict to the canonical identification of tangent spaces $\left.\mathbb{R}_{H \mathbb{F}_{2} P}^{1}=\widehat{\mathbb{A}}^{1,(1)}\right)$.

Proof The conclusion of the previous corollary is that the $\mathbb{F}_{2}$-points of Spec $H \mathbb{F}_{2} P_{0}(M O)$ biject with classes in $H \mathbb{F}_{2} P^{0} M O(1) \cong \widetilde{H \mathbb{F}_{2} P} \mathbb{R P}^{\infty}$ satisfying the condition that they give an isomorphism $\mathbb{R P}_{H \mathbb{F}_{2} P}^{\infty} \cong \widehat{\mathbb{G}}_{a}$. Because $H \mathbb{F}_{2} P_{0}(M O)$ is a polynomial algebra, this holds in general: For $u: \mathbb{F}_{2} \rightarrow T$ an $\mathbb{F}_{2}$-algebra, the $T$-points of Spec $H \mathbb{F}_{2} P_{0}(M O)$ will biject with coordinates on $u^{*} \mathbb{R P}_{H \mathbb{F}_{2} P}^{\infty}$. The isomorphism of schemes follows, though we have not yet discussed equivarience.

To compute the action of Aut $\widehat{\mathbb{G}}_{a}$, we turn to the map in Example 1.1.3:

$$
\Sigma^{\infty} B O(1) \stackrel{c, \simeq}{\longrightarrow} \Sigma M O(1) .
$$

Writing $\alpha(t)=\sum_{j=0}^{\infty} \alpha_{j} t^{j}$ and $\xi(t)=\sum_{k=0}^{\infty} \xi_{k} t^{2^{k}}$, the dual Steenrod coaction on $H \mathbb{F}_{2} P_{0} B O(1)$ is encoded by the formula

$$
\sum_{j=0}^{\infty} \psi\left(\alpha_{j}\right) t^{j}=\psi(\alpha(t))=\alpha(\xi(t))=\sum_{j=0}^{\infty} \alpha_{j}\left(\sum_{k=0}^{\infty} \xi_{k} t^{2^{k}}\right)^{j} .
$$

Because $c_{*}\left(\alpha_{j}\right)=\alpha_{j-1}^{\prime}$, this translates to the formula $\psi\left(\alpha^{\prime}(t)\right)=\alpha^{\prime}(\xi(t))$, where

$$
\alpha^{\prime}(t)=\sum_{j=0}^{\infty} \alpha_{j}^{\prime} t^{j+1}
$$


Passing from $H \mathbb{F}_{2} P_{0}(M O(1))$ to

$$
H \mathbb{F}_{2} P_{0}(M O) \cong \operatorname{Sym} H \mathbb{F}_{2} P_{0}(M O(1)) /\left(\alpha_{0}^{\prime}=1\right),
$$

we see that this is precisely the formula for precomposing a coordinate with a strict automorphism. This is exactly the assertion that a point in $\operatorname{Aut}_{1}\left(\widehat{\mathbb{G}}_{a}\right)$ acts on a point in Coord $\left(\mathbb{R P}_{H \mathbb{F}_{2} P}^{\infty}\right)$ in the way claimed.

We are now ready to analyze the group cohomology of $\underline{\operatorname{Aut}}\left(\widehat{\mathbb{G}}_{a}\right)$ with coefficients in the comodule $H \mathbb{F}_{2} P_{0}(M O)$. This is the last piece of input we need to assess the Adams spectral sequence computing $\pi_{*} M O$.

Theorem 1.5.6 ([Str06, theorem 12.2], [Mit83, proposition 2.1]) The action of $\underline{A u t}_{1}\left(\widehat{\mathbb{G}}_{a}\right)$ on $\operatorname{Coord}_{1}\left(\widehat{\mathbb{G}}_{a}\right)$ is free:

$$
\operatorname{Coord}_{1}\left(\widehat{\mathbb{G}}_{a}\right) \cong \operatorname{Spec} \mathbb{F}_{2}\left[b_{j} \mid j \neq 2^{k}-1\right] \times \underline{\operatorname{Aut}}_{1}\left(\widehat{\mathbb{G}}_{a}\right) .
$$

Proof Recall, again, that $\underline{\mathrm{Aut}}_{1}\left(\widehat{\mathbb{G}}_{a}\right)$ is defined by the (split) kernel sequence

$$
0 \rightarrow \underline{\text { Aut }}_{1}\left(\widehat{\mathbb{G}}_{a}\right) \rightarrow \underline{\text { Aut }}\left(\widehat{\mathbb{G}}_{a}\right) \rightarrow \mathbb{G}_{m} \rightarrow 0
$$

Consider a point $f \in \operatorname{Coord}_{1}\left(\widehat{\mathbb{G}}_{a}\right)(R)$, which in terms of the standard coordinate can be expressed as

$$
f(x)=\sum_{j=1}^{\infty} b_{j-1} x^{j},
$$

where $b_{0}=1$. Decompose this series as $f(x)=f_{\text {typ }}(x)+f_{\text {rest }}(x)$, with

$$
f_{\text {typ }}(x)=\sum_{k=0}^{\infty} b_{2^{k}-1} x^{2^{k}}, \quad f_{\text {rest }}(x)=\sum_{j \neq 2^{k}} b_{j-1} x^{j} .
$$

Because we assumed $b_{0}=1$ and $f_{\text {typ }}$ is concentrated in power-of- 2 degrees, it follows that $f_{\text {typ }}$ gives a point $f_{\text {typ }} \in \underline{\text { Aut }}_{1}\left(\widehat{\mathbb{G}}_{a}\right)(R)$. We can use it to de-scale and get a new coordinate

$$
g(x)=f_{\text {typ }}^{-1}(f(x))=f_{\text {typ }}^{-1}\left(f_{\text {typ }}(x)\right)+f_{\text {typ }}^{-1}\left(f_{\text {rest }}(x)\right),
$$

which itself has an analogous decomposition into series $g_{\text {typ }}(x)$ and $g_{\text {rest }}(x)$. Finally, note that $g_{\text {typ }}(x)=x$, since $f_{\text {typ }}^{-1}\left(f_{\text {rest }}(x)\right)$ cannot contribute to the powerof-2 degrees in the composite, and that $f_{\text {typ }}$ is the unique point in $\underline{\mathrm{Aut}}_{1}\left(\widehat{\mathbb{G}}_{a}\right)(R)$ that has this property. Altogether, this shows that the map $f \mapsto\left(f_{\text {typ }}, g_{\text {rest }}\right)$ is an isomorphism.

Corollary 1.5.7 ([Str06, remark 12.3]) $\pi_{*} M O=\mathbb{F}_{2}\left[a_{j} \mid j \neq 2^{k}-1, j \geq 1\right]$ with $\left|a_{j}\right|=j$. 
Proof Set $M=\mathbb{F}_{2}\left[a_{j} \mid j \neq 2^{k}-1\right]$, and write $\mathcal{A} P_{0}^{\prime}$ for the ring of functions on $\underline{A u t}_{1}\left(\widehat{\mathbb{G}}_{a}\right)$. It follows from Corollary 1.4.16 applied to Theorem 1.5.6 that the $\underline{A u t}_{1}\left(\widehat{\mathbb{G}}_{a}\right)$-cohomology of $H \mathbb{F}_{2} P_{0}(M O)$ has amplitude 0 :

$$
\begin{aligned}
\operatorname{Cotor}_{\mathcal{A} P_{0}^{\prime}}^{*, *}\left(\mathbb{F}_{2}, H \mathbb{F}_{2} P_{0}(M O)\right) & =\operatorname{Cotor}_{\mathcal{A} P_{0}^{\prime}}^{* * *}\left(\mathbb{F}_{2}, \mathcal{A} P_{0}^{\prime} \otimes_{\mathbb{F}_{2}} M\right) \\
& =\mathbb{F}_{2} \square_{\mathcal{A} P_{0}^{\prime}}\left(\mathcal{A} P_{0}^{\prime} \otimes_{\mathbb{F}_{2}} M\right) \\
& =\mathbb{F}_{2} \otimes_{\mathbb{F}_{2}} M=M .
\end{aligned}
$$

Since the Adams spectral sequence

$$
H_{\mathrm{gp}}^{*}\left(\underline{\mathrm{Aut}}_{1}\left(\widehat{\mathbb{G}}_{a}\right) ; H \mathbb{F}_{2} P_{0}(M O)\right) \Rightarrow \pi_{*} M O
$$

is concentrated on the 0 -line, it collapses. We can infer the grading from the residual $\mathbb{G}_{m}$-action:

$$
f_{\text {rest }}(x) \mapsto \xi_{0}^{-1} f_{\text {rest }}\left(\xi_{0} x\right)=\sum_{j \neq 2^{k}} \xi_{0}^{j-1} a_{j-1} x^{j} .
$$

We therefore deduce

$$
\pi_{*} M O=\mathbb{F}_{2}\left[a_{j} \mid j \neq 2^{k}-1\right] .
$$

This is pretty remarkable: Some statement about manifold geometry came down to understanding how we could reparameterize a certain formal group, itself a (fairly simple) purely algebraic problem. The connection between these two problems seems fairly miraculous: We needed a small object, $\mathbb{R} \mathrm{P}^{\infty}$, which controlled the whole story; we needed to be able to compute everything about it; and we needed various other "generation" or "freeness" results to work out in our favor. It is not obvious that we will get this lucky twice, should we try to reapply these ideas to other cases. Nevertheless, trying to push our luck as far as possible is the main thrust of the rest of the book. We could close this section with this accomplishment, but there are two easy consequences of this calculation that are worth recording before we leave.

Lemma 1.5.8 $M O$ splits as a wedge of shifts of $\mathrm{HF}_{2}$.

Proof Referring to Lemma 1.4.2, we find that the Hurewicz map induces a $\pi_{*}$-injection $M O \rightarrow H \mathbb{F}_{2} \wedge M O$. Pick an $\mathbb{F}_{2}$-basis $\left\{v_{\alpha}\right\}_{\alpha}$ for $\pi_{*} M O$ and extend it to an $\mathbb{F}_{2}$-basis $\left\{v_{\alpha}\right\}_{\alpha} \cup\left\{w_{\beta}\right\}_{\beta}$ for $\pi_{*} H \mathbb{F}_{2} \wedge M O$. Altogether, this larger basis can be represented as a single map,

$$
\bigvee_{\alpha} \Sigma^{\left|v_{\alpha}\right|} \mathbb{S} \vee \bigvee_{\beta} \Sigma^{\left|w_{\beta}\right|} \mathbb{S} \stackrel{\bigvee_{\alpha} v_{\alpha} \vee \bigvee_{\beta} w_{\beta}}{\longrightarrow} H \mathbb{F}_{2} \wedge M O
$$


Smashing through with $H \mathbb{F}_{2}$ gives an equivalence

$$
\bigvee_{\alpha} \Sigma^{\left|v_{\alpha}\right|} H \mathbb{F}_{2} \vee \bigvee_{\beta} \Sigma^{\left|w_{\beta}\right|} H \mathbb{F}_{2} \stackrel{\simeq}{\rightarrow} H \mathbb{F}_{2} \wedge M O
$$

The composite map

$$
M O \rightarrow H \mathbb{F}_{2} \wedge M O \stackrel{\simeq}{\longleftarrow} \bigvee_{\alpha} \Sigma^{\left|v_{\alpha}\right|} H \mathbb{F}_{2} \vee \bigvee_{\beta} \Sigma^{\left|w_{\beta}\right|} H \mathbb{F}_{2} \rightarrow \bigvee_{\alpha} \Sigma^{\left|v_{\alpha}\right|} H \mathbb{F}_{2}
$$

is a weak equivalence.

Remark 1.5.9 Just using that $\pi_{*} M O$ is connective and $\pi_{0} M O=\mathbb{F}_{2}$, we can produce a ring spectrum map $M O \rightarrow H \mathbb{F}_{2}$. What we have learned is that this map has a splitting: Lemma 1.5 .8 can be used to factor the unit map of $M O$ through $\mathrm{HF}_{2}$ in a way that makes it an $H \mathbb{F}_{2}$-algebra.

Remark 1.5.10 We are also in a position to understand the stable cooperations $M O_{*} M O$. We may rewrite this as

$$
\begin{aligned}
M O_{*} M O & =\pi_{*} M O \wedge M O=\pi_{*} M O \wedge_{H \mathbb{F}_{2}}\left(H \mathbb{F}_{2} \wedge M O\right) \\
& \Leftarrow \operatorname{Tor}_{*, *}^{\mathbb{F}_{2}}\left(M O_{*}, H \mathbb{F}_{2 *} M O\right)=M O_{*} \otimes_{\mathbb{F}_{2}} H \mathbb{F}_{2 *} M O .
\end{aligned}
$$

Hence, a point in $\operatorname{Spec} M O_{*} M O$ consists of a pair of points in $\operatorname{Spec} M O_{*}$ and $\operatorname{Spec} H \mathbb{F}_{2 *} M O$, which we have already identified respectively as formal group laws with vanishing 2-series, a property, and formal group laws with specified logarithms, data. This description can be amplified to capture all of the structure maps: A formal group law with vanishing 2-series admits a logarithm, which indicates how the composition and conjugation maps of Definition 3.1.13 behave. 
